\title{
Las labiadas de la expedición Malaspina (1789-1794). I
}

\author{
José Luis Fernández-Alonso y Ramón Morales ${ }^{1}$
}

Resumen: Fernández-Alonso, J. L. \& Morales, R. 2013. Las labiadas de la expedición Malaspina (1789-1794). I. Bot. Complut. 37: 153-169.

Se estudiaron e identificaron los pliegos de la familia Lamiaceae depositados en el herbario MA recolectados por Luis Neé durante la expedición Malaspina, la cual duró algo más de 5 años y recorrió gran parte de América, Filipinas, Australia y algunas islas del océano Pacífico. En total se estudiaron 166 pliegos correspondientes a 68 especies y 23 géneros de labiadas. La mayoría de las colecciones identificadas (14 géneros y 51 especies) proviene de América. Se ha localizado nuevo material tipo de dos especies del género Gardoquia. Se ha excluido el género Salvia (otros 164 pliegos correspondientes a 46 especies), ya que será publicado en un trabajo independiente posterior.

Palabras clave: América, Australia, Expedición Malaspina, Filipinas, Herbario MA, Lamiaceae, siglo XVIII.

Abstract: Fernández-Alonso, J. L. \& Morales, R. 2013. The labiate family in the Malaspina expedition (1789-1794). I. Bot. Complut. 37: 153-169.

The sheets of Lamiaceae deposited in the herbarium MA collected by Luis Neé during the Malaspina expedition, that in 5 years traveled along America, Philippines, Australia, and some Pacific islands. They were studied and identified 166 sheets corresponding to 68 species and 23 genera of the Lamiaceae family. The most of the identified collections (14 genera and 51 species) are from América. New type material has been located of two species of Gardoquia. In a subsequent independent work the genus Salvia (another 164 sheets corresponding to 46 species) will be published.

Key words: America, Australia, Herbarium MA, Lamiaceae, Malaspina expedition, $18^{\text {th }}$ century, Philippines.

\section{INTRODUCCIÓN}

La expedición Malaspina fue un largo viaje de dos corbetas, la Descubierta y la Atrevida, al mando de Alejandro Malaspina y de José Bustamante respectivamente, a través del océano Atlántico y el océano Pacífico. Se trató de un viaje científico y político. Fondearon en la mayoría de los puertos americanos atlánticos y pacíficos, desde Montevideo hasta Alaska. Y después en las islas Marianas, las Filipinas, Macao en China, Australia, Nueva Zelanda, las Islas Tonga y vuelta por América a España. Parten de Cádiz a mediados de 1789, durante el reinado de Carlos III y regresan en septiembre de 1794, después de más de 5 años de viaje, cuando ya reinaba Carlos IV.

Los resultados de la expedición fueron notables: más de 200 cartas marinas levantadas, estudios de Ciencias Naturales relativos a Botánica, Zoología, Antropología y Geología, así como obtención de datos sobre la situación política y económica de lo que entonces era el imperio español en América y Asia. Los trabajos referentes a la
Botánica fueron realizados por Luis Neé y Tadeo Haencke, aunque de este último no se conserva material en Madrid. Luis Neé recolectó gran número de especímenes, los cuales desafortunadamente fueron incluidos en el herbario general del Real Jardín Botánico de Madrid antes de haber sido estudiados con detalle. Ello acarreó que algunas etiquetas se perdieran o cambiaran en el proceso, 10 que se ha detectado al encontrar especies que no correspondían con la localidad que se cita en la etiqueta o muestras que incluían dos etiquetas de regiones diferentes. Algunas de ellas fueron transcritas, de forma imprecisa, posteriormente.

Debido a que los trabajos sobre el herbario recolectado en la expedición fueron abandonados, dadas las circunstancias políticas e históricas de los últimos años del siglo XVIII y comienzos del XIX (Muñoz Garmendia, 1992), algunos nombres asignados por Neé a especies nuevas, y que aparecen en las etiquetas de sus ejemplares, nunca fueron publicados. Algunos de estos nombres, a los que debería añadirse la locución «ex Neé», fueron utilizados

\footnotetext{
${ }^{1}$ Real Jardín Botánico, CSIC, Plaza de Murillo 2, 28014-Madrid, España.

Sirva este trabajo como homenaje a una maestra y colega, Maruja Carrasco, convencida de la importancia de las colecciones de plantas 0 herbarios.

Recibido: 27 febrero 2013. Aceptado: 8 marzo 2013.
} 
sin la misma por otros autores al describir dichas especies con posterioridad, incluso sin hacer referencia explícita a Neé, quien había recolectado el material de las nuevas especies y asignado un nombre a las mismas (in sched.). En este trabajo se estudiaron los pliegos de la familia de las Labiadas encontrados hasta ahora en el herbario del Real Jardín Botánico de Madrid (MA), 166 pliegos correpondientes a 23 de los 24 géneros identificados. Las colecciones pertenecientes al extenso género Salvia (164 pliegos, pertenecientes a 46 especies), todas americanas, serán tratadas en un trabajo independiente posterior.

Los herbarios siguen siendo una fuente inagotable de información morfológica, geográfica, ecológica y etnobotánica, y como tal, una pieza fundamental en los estudios taxonómicos y florísticos que son los que sostienen la botánica sistemática. Las colecciones botánicas de la Expedición de Malaspina, conservadas desde hace más de dos siglos, son un fiel testimonio del valor implícito que representa este tipo de información y de su importancia como almacén científico dentro de esta área del conocimiento.

\section{METODOLOGÍA}

Se seleccionaron y revisaron los pliegos correspondientes a la familia Lamiaceae (excluido Salvia), que fueron recolectados durante la expedición Malaspina en América, Filipinas y Australia. Los pliegos se encuentran incluidos actualmente en la colección del herbario general del Real Jardín Botánico de Madrid (MA). El recolector de las plantas conservadas en el herbario MA, salvo raras excepciones, fue Luis Neé, quién participó como botánico en dicha expedición.

En base a estas colecciones, algunos botánicos hispanos contemporáneos como Casimiro Gómez Ortega, Antonio José Cavanilles, Mariano Lagasca y el propio Neé describieron un número importante de especies nuevas, aspecto que quedó bien documentado en Muñoz Garmendia (1992). Los pliegos correspondientes a éstas se conservan en la colección de tipos de Cavanilles 0 en el herbario general en MA.

Se identificaron los pliegos estudiados y se actualizaron los nombres conforme a la nomenclatura vigente. Los taxones se presentan por orden alfabético de género y especie, indicando sus autores así como la cita de la publicación original. Se incluyen también algunos sinónimos. De cada uno de los pliegos estudiados se indica el número, si hay duplicado(s), si está en flor (fl) 0 fruto (fr), se transcriben las etiquetas antiguas [et. ant.], manuscritas en general a tinta, con algunos números a lápiz. Después de la localidad y cuando se conoce, se ha intercalado entre corchetes la fecha de recolección (Muñoz Garmendia, 1992). A continuación se incluye también el texto de la etiqueta transcrita [et. tra.] posteriormente, que a veces incorpora información adicional. Cuando no hay etiqueta antigua, se indica expresamente. Al final se incluye el nombre y fecha del especialista que ha identificado el material, según consta en la etiqueta de revisión. En los casos en los que existen láminas realizadas por los dibujantes de la expedición o bien publicaciones de especies relacionadas, se indican también, las citas correspondientes. Finalmente se aportan observaciones y datos relativos a la distribución general de cada especie.

Se señalan los casos en los cuales se ha detectado etiquetado incorrecto o bien mezcla de etiquetas de dos localidades geográficas diferentes asociadas a una sola muestra. En todos los casos se efectuaron las aclaraciones consideradas oportunas.

\section{RESULTADOS}

Para este trabajo se estudiaron 166 pliegos correspondientes a 68 especies de Labiadas ubicadas en 23 géneros (excluido el género Salvia). El material estudiado fue recolectado en las islas Filipinas, las islas Marianas, Australia, Canadá (Nutka), México, Panamá, Ecuador, Perú, Chile, Argentina y Uruguay. Para cada género se incluyen datos relativos al número de especies que lo conforman, así como su distribución general. Para cada especie se indica su distribución y la información asociada a los pliegos revisados incluyendo el número del herbario MA. Buena parte de las muestras estudiadas contaba solo con identificación a nivel de familia o bién con la identificación genérica que fue dada por los botánicos de la expedición, la cual en muchos casos hacía solo referencia a géneros subcosmopolitas o a otros cuya distribución se restringe hoy al viejo Mundo (Bystropogon L'Her., Dracocephalum L., Hyssopus L., Horminum L., Leonurus L., Mentha L., Phlomis L., Sideritis L.y Thymus L.).

Labiadas de Asia-Australia. Entre los ejemplares de la familia Lamiaceae recolectados por los expedicionarios, las colecciones de Asia y Australia contienen especímenes de 10 géneros entre los que destacan algunos de amplia distribución en zonas tropicales del Viejo Mundo como Ajuga L. (2 sp.), Ocimum L. (1 sp.), Leucas R. Br. (3 spp.) y Pogostemon Desf. (1 sp.). Las tres especies del género Leucas provienen de las Filipinas, aunque en la etiqueta de una de las muestras (L. marrubioides Desf.) por error se indica que fue recogida en Perú. Entre los géneros paleotropicales con distribución más restringida se encuentran Anisomeles R. Br. y Basilicum Moench. de Asia y África y Westringia Sm., endémico de Australia. Cabe destacar que entre los ejemplares recolectados en el Paleotrópico y en Australia ya se encuentran algunas plantas introducidas provenientes de Europa, África o América. En la mayoría de los casos, dichas especies son hoy bien co- 
nocidas como malas hierbas de zonas tropicales o como plantas cultivadas desde hace mucho tiempo por el hombre. Este es el caso de las especies americanas Hyptis suaveolens (L.) Poit. e H. spicigera Lam., que ya estaban presentes en Filipinas en la época de la expedición y el de Prunella vulgaris L., planta europea recolectada por aquellas fechas también en Australia. Esta especie se dispersa con facilidad en zonas con prados y cultivos abandonados. Entre las cultivadas se encuentra Plectranthus rotundifolius (Poiret) Sprengel, oriunda de Africa y muy apreciada por sus tubérculos comestibles. Es la llamada «patata de los hausas», etnia que vive en la franja saheliana de África occidental. Ocimum americanum L. es una especie de albahaca oriunda de África tropical y de Asia. Posiblemente se cultivaba ya en Filipinas.

Labiadas americanas. Con diferencia, la mayor diversidad se presenta en las colecciones americanas, representadas por 14 géneros. Destacan entre los de amplia distribución, aparte de Salvia L. (44 especies): Stachys L. (8) incluyendo una recolectada en Canadá, Scutellaria L. (4) y Teucrium L. (3). Entre los géneros con distribución restringida al neotrópico se encuentran Hyptis con 16 especies recolectadas en diferentes lugares de América, Gardoquia Ruiz \& Pav. (7 spp.) y Lepechinia Willd. (5 spp.) predominantemente sudamericanos. Cabe destacar además a los géneros Agastache J. Calyton ex Gronov., Asterohyptis Epling, Cunila L. y Poliomintha A. Gray, que cuentan con representantes en México y Mesoamérica, y a Glechon Spreng. y Minthostachys Griseb., representados solo en Sudamérica. En el caso de Hedeoma Pers., con distribución disyunta en ambos subcontinentes, entre las colecciones de la expedición se encuentra una especie de México y otra de Argentina. La presencia de un pliego de la especie exclusivamente colombiana Lepechinia salviifolia (Kunth) Epling., atribuido a Neé, seguramente obedece a una confusión en las estiquetas, en los trasiegos de muestras de herbario en el Real Jardín Botánico, ya que Neé no estuvo en Colombia. Este pliego actualmente incluido en el herbario general en MA, tiene que ver con uno de los escasos envíos de pliegos y semillas efectuados por José Celestino Mutis a Linneo y al Real Jardín antes del inicio formal de la Expedición del Nuevo Reino de Granada en 1783 (Fernández Alonso \& Amaya 1991, Amaya 2005).

El orégano Origanum x majoricum Cambess., es una especie híbrida entre 0 . vulgare L. y 0 . majorana L., la cual se cultiva actualmente sobre todo en la isla de Mallorca, pero que está presente en otros países de América como Colombia y Ecuador, siendo una planta habitual en los mercados de plantas aromáticas y medicinales (Fer- nández Alonso \& Rivera 2006). La muestra de este orégano híbrido recolectada por Neé proviene concretamente de Perú, donde sorprende su presencia tan temprana. Hay que resaltar no obstante, que las plantas aromáticas de origen euroasiático, fueron intensamente comercializadas y cultivadas en las colonias, donde muchas de ellas eran utilizadas con regularidad en las farmacopeas (Díaz \& Mantilla 2002, Morales 2010, Díaz 2012).

Especies nombradas por Neé in schaedis. Entre las plantas recolectadas por Neé durante la expedición Malaspina, hubo muchas que el alcanzó a considerar como nuevas especies, hecho que constató con su puño y letra en las etiquetas de herbario. Dentro del grupo ahora abordado encontramos cuatro especies del género Gardoquia que realmente nadie había nombrado cuando Neé les asignó un nombre de especie nueva en la etiqueta (in schaed.). La planta chilena Gardoquia linearis Neé sp. n., fue descrita formalmente en 1832 como G. chilensis por Bentham utilizando una colección posterior proveniente de Valparaíso (Lay \& Collie s.n., K). La Gardoquia rotundifolia Neé n. sp., recolectada en Perú y Ecuador, sería formalmente nombrada por Kunth en 1818 como G. tomentosa. La planta mexicana Gardoquia cordifolia Neé, sp. nov., sería descrita por Bentham en 1840 como G. mexicana. Por último, la especie peruana Gardoquia sericea Neé $\mathrm{n}$. sp., sería publicada por Bentham en 1834, a partir de una planta nombrada por C. C. Presl con el mismo nombre. Parece verosimil que el origen de este nombre esté en una de las colecciones anotadas de Neé, que fueron incluidas en el herbario de T. Haenke (Presl 1825-35). Lo que se ha conservado del herbario de Haenke, fue a parar al Museo bohémico de Praga, donde fue estudiado y publicado parcialmente por K. B. Presl (Ibañez Montoya 1992, Muñoz Garmendia 1992, 2001). El grupo más numeroso de colecciones de Haenke esta depositado hoy en el herbario PR, en Praga.

\section{Lista de especies}

1. Ajuga L., consta de unas 40 especies que viven sobre todo en Eurasia, con su mayor diversidad hacia el este de la misma (Cantino in Harley et al. 2004: 201).

1.1. Ajuga australis R. Br., Prodr. Fl. Nov. Holland.: 503 (1810). Planta de Australia.

MA 256903 fl, fr: [AUSTRALIA] «Teucrium / s. n / ex Nova Hollandia [III a IV-1793] / Neé dedit.» [et. ant.]; «Teucrium / s. n / ex Nova Holanda / Neé dedit (Exp. Mal.)» [et. tra.]. Det. R. Morales, VIII-2012. 
1.2. Ajuga taiwanensis Nakai ex Murata, Acta Phytotax. Geobot. 23: 23 (1968). Planta de Filipinas.

MA 254611, fl (2 pliegos): [FILIPINAS] «Ajuga orientalis [III a XII-1792] / Neé dedit» [et. ant.]; «Ajuga orientalis / Neé dedit» [et. tra.]. Det. R. Morales, III-2012.

2. Agastache J. Clayton ex Gronov., consta de unas 22 especies que viven en el norte de América y una en Asia (Budantsev in Harley et al. 2004: 251).

2.1. Agastache mexicana (Kunth) Lint. \& Epling, Amer. Midl. Naturalist 33: 227 (1945). Planta de México.

Dracocephalum mexicanum Kunth, MA 254943, fl: [MÉ$\mathrm{XICO}$ «Dracoceph. / Iuxta Atotonilco el gran- / de en Novahispania. [3 a 4-IX-1791] / Neé dedit» [et. ant.]; Dracocephalum / Atotonilco el grande en Nueva España / Cavanilles / Neé dedit» [et. tra.]. Det. R. Morales, III-2002. MA 254974, fl, fr: [MÉXICO] «Gardoquia / prope Guanajuato in No- / va Hispania. [19 a 23-X-1791] / Neé dedit,» [et. ant.]; «Gardoquia?/ próximo a Guanajuato en Nueva España / Cavanilles / Neé dedit (Exp. Mal.)» [et. tra.]. Det. R. Morales, III-2002. MA 254976, fl, fr: [MÉXICO] «Gardoquia / Ex Chalma [hacia 24-VIII-1791] / Neé iter»« [et. ant.]; «Gardoquia / Chalma / J. D. Rodríguez / Neé iter (Exp. Mal.),» [et. tra.]. Det. R. Morales, VIII-2002. MA 254977, fl: [MÉXICO] «Gardoquia / Ex Pachuca et Real del monte / in Nov. Hispania [27 a 30-VIII-1791] / Neé iter» [et. ant.]; «Gardoquia / Pachuca y Real del Monte en Nueva España / Neé iter (Exp. Mal.),» [et. tra.]. Det. R. Morales, VIII-2002.

3. Anisomeles R. Br., consta de 3 especies que viven en África tropical, sur de Asia y nordeste de Australia (Ryding in Harley et al. 2004: 217).

3.1. Anisomeles indica (L.) Kuntze, Rev. Gen. Pl. 2: 512 (1891). Planta del paleotrópico.

Nepeta indica L.; Epimeredi indicus (L.) Rothm.; Marrubium indicum (L.) Burm. fil. MA 256672, fl: [FILIPINAS] «Stachys / ex Philippicis insulis [III a XII-1792] / Neé iter» [et. ant.]; «Stachys / Islas Filipinas / J. D. Rodríguez / Neé iter (E.M.)» [et. tra.]. Det. J. L. Fern. Alonso, II-2013. MA 255004, fr: [FILIPINAS] «Horminum / Del Perú / Neé iter» [et. ant.]; «Horminum / Perú / Neé iter (Exp. Mal.)» [et. tra.]. Det. J. L. Fern. Alonso, I-2013.

Dada la correspondencia morfológica entre las dos muestras de esta especie tan característica y el hecho de que no se encuentre en América, consideramos un error la indicación «del Perú» en una de las muestras.

4. Asterohyptis Epling., consta de 3 especies que viven desde México hasta Costa Rica (Harley in Harley et al. 2004: 201).
4.1. Asterohyptis mociniana (Benth.) Epling, Bull. Torrey Bot. Club 60: 19 (1933). Planta de México.

Hyptis mociniana Benth., Lab. Gen. Sp.: 129 (1833); Hyptis alopecuroides Briq.; Hyptis spinulosa Benth.; Hyptis biolleyi Briq. MA 101056, fl, fr: [MÉXICO]«Sideritis [1791] / Neé iter» [et. ant.]; «Sideritis / Neé iter / 101056» [et. tra.]. Det. J. L. Fern. Alonso, II-2013.

4.2. Asterohyptis stellulata (Benth.) Epling, Bull. Torrey Bot. Club 60: 17 (1933). Planta de México.

Hyptis stellulata Benth., Lab. Gen. Sp: 129 (1833); Hyptis pubescens Benth.; Mesosphaerum stellulatum (Benth.) Kuntze; MA 254651 (177683 es el número antiguo), fl: [MÉXICO] «iBudleia / Acapulco. [1791] / Neé dedit.» [et. ant.]; «iBudleia / Acapulco. / Neé dedit / 177683» [et. tra.]; //Labiatae / non Buddleja. Det. A. J. M. Leeuwenberg, 1973 (WAG)/ Det. R. Harley, X-2005. MA 101057 fl, fr: [MEXICO] «iSideritis / ex Nov. Hispania et Peruvia [1791] / Neé dedit.» [et. ant.]; «iSideritis / ex nov. Hispania et Peruvia / Neé iter» [et. tra.]. Det. J. L. Fern. Alonso, II-2013.

5. Basilicum Moench., consta de una sola especie que vive en los trópicos del Viejo Mundo, con frecuencia en lugares estacionalmente encharcados (Paton in Harley et al. 2004: 262).

5.1. Basilicum polystachyon (L.) Moench, Suppl. Meth. P1.: 143 (1802).

Ocimum polystachyon L., Mant. Pl.: 567 (1771); Moschosma polystachyon (L.) Benth.; Ocimum tenuiflorum Burm. f., non L. MA 255427, fl: [FILIPINAS] «Ocymum? / ex insulis philippicis [III a XII-1792] / Neé iter» [et. ant.]; «Ocymum? / Islas Filipinas / Neé iter Salvia / Neé iter (Exp. Mal.)» [et. tra.]. Det. R. Morales, II-2013. MA 255428, fl: [FILIPINAS] «¿Ocymum / In insulis philippicis [III a XII-1792] / Neé dedit» [et. ant.]; «Ocymum? / Islas Filipinas / Cavanilles / Neé dedit (Exp. Mal.)» [et. tra.]. Det. R. Morales, II-2013.

6. Cunila L., consta de unas 15 especies americanas que viven en el sur de Norteamérica, Centroamérica y el Cono Sur (Cantino in Harley et al. 2004: 246).

6.1. Cunila polyantha Benth., Lab. Gen. Sp.: 362 (1834).

MA 255294 (2 pliegos), fl: [MÉXICO] «Pachuca. Nueva España. [27 a 29-VIII-1791] / Neé iter (Exp. Mal.),» [et. tra.]. Det. R. Morales, IV-2012.

7. Gardoquia Ruiz \& Pav., Cantino \& Harley (Cantino in Harley et al. 2004: 241) incluyen este género dentro de Clinopodium. En nuestra opinión es un desatino. Clino- 
podium, cuya especie tipo es $C$. vulgare, se diferencia bien por sus caracteres genéricos de cualquier especie de Gardoquia. Este género americano consta de unas 40 especies que viven desde México hasta Chile (Epling \& Játiva 1964). Entre los especímenes recolectados en la expedición se encuentra una especie mexicana y otras 6 sudamericanas. Cuatro de ellas fueron anotadas por Neé como especies nuevas, aspecto que se comenta arriba.

7.1. Gardoquia gilliesii Graham, Edinburgh New Philos. J.: 377 (1831). Planta de Chile.

Gardoquia chilensis Benth. in Hook. \& Arn., Bot. Beech. Voy.: 58 (1832); Clinopodium chilense (Benth.) Govaerts; Gardoquia linearis Neé, sp. n., sin descripción (in sched.). MA 254986 fl, fr: «Gardoquia / Chile. / Neé dedit» [et. ant.]; «Gardoquia / Chile. / Cavanilles / Neé dedit (Exp. Mal.)» [et. tra.]. Det. R. Morales, IV-2012. MA 256140 (2 pliegos), fl.: «Gardoquia? / Talcahuano in Chile [XI-1793] / Neé dedit» [et. ant.]; «Ex Ant. Herb. Generali / Satureja sp. « [et. tra.]. Det. R. Morales, IV-2012. MA 256142, fl: [CHILE] «712 / Gardoquia / Neé iter Neé iter» [et. ant.]; «Satureja sp. / Ex. Ant. Herb. Gen.» [et. tra.]. Det. R. Morales, IV-2012. MA 256142/2b: [CHILE] «390 / Gardoquia linearis sp. n. / Ex / Neé iter» [et. ant.]; «Satureja sp. / Ex. Ant. Herb. Gen.» [et. tra.]. Det. R. Morales, IV-2012. MA 256142/3, fl: «Gardoquia / Del Perú y Chile / Neé iter» [et. ant. 1]; «Gardoquia linearis sp. n. / var. / Neé iter» [et. ant. 2]. Det. R. Morales, III-2012. MA 256142/4: «Duraznillo. / Chile» [et. ant. 1]; «Gardoquia / ex N. Hisp. / Neé iter» [et. ant. 2]; «Gardoquia / ex Chile / Neé iter»; «Gardoquia linearis sp. nov. / ex Peruvia / Neé iter» [et. ant. 2]. Det. R. Morales, III-2012.

Gardoquia gilliesii Graham y Micromeria gilliesii Bentham son dos especies bien diferenciadas, aunque Briq. in Engler \& Prantl, Nat. Pflanzenfam. 4(3a): 300 (1896), combinó como Satureja las dos especies: Satureja gilliesii (Benth.) Briq. y Satureja gilliesii (Graham) Briq.; después en la página 380 se corrige diciendo que la primera corresponde con Satureja oligantha Briq. Solo una de ellas corresponde a Gardoquia, por lo que no hay ningun problema nomenclatural.

7.2. Gardoquia tomentosa Kunth in Humb., Bonpl. \& Kunth, Nov. Gen. Sp. 2: 314 (1818). Planta de Perú y Ecuador.

Gardoquia elegans Kunth in Humb., Bonpl. \& Kunth, Nov. Gen. Sp. 2: 315 (1818); Gardoquia grandiflora Kunth in Humb., Bonpl. \& Kunth, Nov. Gen. Sp. 2: 314 (1818); Gardoquia thymoides Kunth in Humb., Bonpl. \& Kunth, Nov. Gen. Sp. 2: 314 (1818); Gardoquia incana Willd. ex Benth., Linnaea 11: 331 (1837); Gardoquia quitensis Willd. ex Benth., Linnaea 11: 331. 1837; Clinopodium tomentosum (Kunth) Harley; MA 254972, f1.: [PERÚ] «Gardoquia rotundifolia / sp. nov. / Neé iter» [et. ant.]; «Gardoquia rotundifolia / sp. nov. / J. D. Rodrí- guez/ Neé iter (Exp. Mal.?)» [et. tra.]. Det. R. Morales, II-2013. MA 254979: [ECUADOR] «¿Gardoquia / inter Guaranda et montem / Chimborazo [4 a 21-X-1790] / Neé dedit« [et. ant.]; ¿Gardoquia / Entre Guaranda et montem / Chimborazo / Cavanilles / Neé dedit (Exp. Mal.)» [et. tra.]. Det. R. Morales, XI1988. MA 256138: «Perú / San Buenaventura [6 a 18-VII-1790] / Gardoquia» [et. ant.]; «Satureja / Ex. Ant. Herb. Gener.» [et. tra.]. Det. R. Morales, II-2013.

7.3. Gardoquia incana Ruiz \& Pav., Syst. Veg.: 148 (1789). Planta de Perú y Chile.

Satureja incana (Ruiz \& Pav.) Spreng.; Satureja pavoniana Briq.; Gardoquia breviflora Benth.; Clinopodium breviflorum (Benth.) Govaerts; MA 254970A, fl: «Gardoquia incana / Fl. Per. / Chile / Neé dedit» [et. ant.]; «Gardoquia incana? / Fl. Peruv. / Chile / Cavanilles / Neé dedit (Exp. Mal.)» [et. tra.]. Det. R. Morales, II-2013. MA 254985 fl: «Gardoquia / ex Chile. Neé iter» [et. ant.]; «Gardoquia / Chile. / Cavanilles / Neé iter (Exp. Mal.)» [et. ant.]. Det. R. Morales, II-2012. MA 256131 fl: [PERÚ] «Gardoquia / Del Perú [V a IX-1790] / Neé iter» [et. ant.]; «Satureja pavoniana Briq.?» [et. tra.]. Det. R. Morales, X-1990. MA 256131/2, est.: [PERÚ] «Gardoquia obovata ? Fl. Perú/ Del Perú y Panamá / Neé iter» [et. ant.]; «Satureja pavoniana Briq.?» [et. tra.]. Det. R. Morales, X-1990. MA 256131/3 fl,; [PERÚ] «Gardoquia incana Fl. P. / ex Pachuca in nov. Hispania / Neé iter» [et. ant. 1]; «Gardoquia incana Syst. Veg / Flor. Peruv. / Ex Peruvia [V a IX-1790] / Neé dedit anno 1801» [et. ant. 2]; «Satureja pavoniana Briq.» [et. tra.]. Det. R. Morales, X-1990.

En todos los casos se trata de plantas procedentes de Perú y Chile. Las indicaciones «Panamá» y «Pachuca in nov. Hispania» que acompañan en dos de los pliegos a las de las localidades correctas, se deben a mezclas de etiquetas en el trasiego de herbarios. En el herbario de Ruiz y Pavón en MA se encuentran tres pliegos catalogados como material tipo de esta especie, que no tienen relación con las colecciones de Neé: (MA 815057, MA 815088 y MA 815099).

7.4. Gardoquia mexicana Benth., Pl. Hartw. (Bentham) Sig. H: 50 (1840). Planta de México.

Satureja mexicana (Benth.) Briq.; Clinopodium mexicanum (Benth.) Govaerts; Gardoquia cordifolia Neé, sp. n., sin descripción (in sched.). MA 254969, fl: [MÉXICO] «Gardoquia cordifolia / sp. nov. / ex nov. Hispania / Neé iter» [et. ant.]; «Gardoquia cordifolia sp. nov. / Nueva España / J. D. Rodríguez / Neé iter (Exp. Mal.)» [et. tra.]. Det. R. Morales \& J. L. Fern. Alonso, II-2013. MA 254975: [MÉXICO] «Gardoquia / en la hacienda de Regla. / Nueva España [3 a 4-IX-1791] / Neé dedit.» [et. ant.]; Gardoquia? / en la hacienda de Regla, / Nueva España / Cavanilles / Neé dedit (Exp. Mal.)» [et. tra.]. Det. R. Morales \& J. L. Fern. Alonso, II-2013. MA 254983: [MÉXICO] «Gardoquia / (colección atribuida Neé)» [et. ant.]; «Gardoquia» [et. tra.]. Det. R. Morales \& J. L. Fern. Alonso, II-2013. 
7.5. Gardoquia multiflora Ruiz \& Pav., Fl. Peruv. 1: 149 (1798). Figs. 1 y 2A. Planta de Perú y Chile.

Satureja multiflora (Ruiz \& Pav.) Briq.; Clinopodium multiflorum (Ruiz \& Pav.) Kuntze; Rizoa ovatifolia Cav., Anales Cienc. Nat. 3(8): 133, tab. 29 (II-1801) [Ind. loc.: «D. Luis Neé halló y mandó dibuxar esta planta en San Carlos de Chiloe por el mes de Febrero. Vila seca y su dibuxo. (Cav., Icon. 6: 56-57, tab. 578 (1801)): «Habitat in San Carlos de Chiloe, floret et fructificat Februario. Vidi siccam et accuratissimam Iconem apud D. Ludovicum Neé.»)]. Chile: San Carlos de Chiloe (Muñoz Garmendia, 1992: 371). Ic.: E. García Guillén \& F. Muñoz Garmendia, 2001: 113, lámina 33. Sign. RJB, VI, lám. 35. La tabla 578 de los Icones de Cavanilles es idéntica a la tabla 29 de los Anales de Ciencias Naturales. MA 29393, f1: [CHILE] «Rizoa ovatifolia / Icon . Tab. 578 / Ex San Carlos de Chiloe [6 a 16-II1790] / Neé dedit.» [et. ant.]; Satureja multiflora R. \& Pav ??» [et. tra.]. Rev. R. Morales \& J. L. Fern. Alonso. MA 254980, fl: [CHILE] «Gardoquia / Neé iter» [et. ant.]; «Gardoquia / Neé iter» [et. ant.]. Det. R. Morales. MA 254981, fl: [CHILE] «Gardoquia / Neé iter» [et. ant.]; «Gardoquia / Neé iter (Exp. Mal.)» [et. tra.]. Det. R. Morales. MA 255542,fl: [CHILE] «Rizoa» [et. ant.]; «Rizoa ovatifolia» (atribuida a Neé) [et. tra.]. Det. R. Morales, 1994. MA 256141-1, fl: [CHILE] «Gardoquia multiflora / Fl. Peruv. / Neé dedit anno 1801.» [et. ant.]; «Satureja / Neé dedit» [et. tra.]. Det. R. Morales \& J. L. Fern. Alonso, II-2013. MA 256141-2, fl: [PERÚ] «Gardochia / Flor. Peruv. / Obragi1lo S Buenaventura [18-VI a 18-VII-1790],» [et. ant. 1]; «Gardoquia elliptica gymnosp. / Fl Per. / F. P. / Perú prope Obragi110 / Neé dedit» [et. ant. 2]; «Gardoquia multiflora / Fl. Peruv. / ex Peruvia / Neé iter» [et. ant. 3]. Det. R. Morales \& J. L. Fernández Alonso, II-2013. MA 655928, fl, fr: [CHILE] «Rizoa ovatifolia / Icon. Tab. 578 / ex San Carlos de Chiloé / Nee dedit / [et. ant.]; «Ex antiquo herbario generali / Satureja multiflora (R. \& P.) Briq. ?? [et. tra.]. Espécimen tipo, colección de Cavanilles: Rev. J. L. Fern. Alonso, II-2013.

7.6. Gardoquia revoluta Ruiz \& Pav., Fl. Peruv. 1: 149 (1798). Planta de Perú.

Satureja revoluta (Ruiz \& Pav.) Briq.; Clinopodium revolutum (Ruiz \& Pav.) Govaerts; MA 254973,fl: [PERÚ] «Gardoquia / ex Nova Hispania» [et. ant. 1]; «Gardoquia Fr. P. / ex Canta in Perú / Neé dedit» [et. ant. 2]; «Gardoquia Fr. P. / Canta en Perú [15-VI a 24-VII-1790]/ Cavanilles / Neé dedit» [et. tra.]. Det. R. Morales, X-1990. MA 256132, fl: [PERÚ] «Gardoquia revoluta. Fl. Peruv. / Del Perú [V a IX-1790] / Neé iter» [et. ant.]; Satureja revoluta (R. \& P.) Briq.» [et. tra.]. Det. R. Morales, X-1990.

7.7. Gardoquia sericea C. Presl ex Benth., Lab. Gen. Sp.: 402 (1834). Planta de Perú y Ecuador.

Satureja sericea (C.Presl. ex Benth.) Briq.; Clinopodium sericeum (C.Presl. ex Benth.) Govaerts; Clinopodium sericeum (C. Presl. ex Benth.) Harley; Gardoquia sericea Neé, sp. n., sin descripción (in sched.). MA 254984, fl: [PERÚ] «Gardochia /
Flor Peruv / Perú» [et. ant. 1]; N. e. Gardochia « [et. ant. 2]; «Gardoquia sericea. sp. nov. / de Perú / Neé iter» [et. tra.]. Det. R. Morales \& J. L. Fern. Alonso, II-2013, como probable Isotypus. MA 256139, f1: [ECUADOR] «Gardoquia / 2» [et. ant. 1]; « G. Gardoquia / en Nueva España / Guayaquil, o Huaiahil» [2 a 27-X-1790] [et. ant. 2]; «Satureja sp.» [et. tra.]. Det. R. Morales \& J. L. Fern. Alonso, II-2013.

La planta peruana nombrada en primera instancia por Luis Neé como «Gardoquia sericea sp. nov.», en la etiqueta de herbario, haciendo referencia al indumento plateado característico de sus tallos y hojas, recibiría muchos años después este mismo nombre en la publicación de $\mathrm{C}$. C. Presl (1925-1935) a partir de otra muestra - probablemente un duplicado de la de Neé-, del coexpedicionario T. Haenke (Ibáñez \& Montoya 1992). El pliego de Haenke, como el resto de su herbario fue a parar a Praga (República Checa), en donde fue estudiado por Presl y, con toda probabilidad tenía ya la anotación de Neé. En el duplicado que actualmente se conserva en en el herbario $\mathrm{K}$ (K193653) y que fué estudiado por Bentham, aparece solo la información transcrita por C. Presl «Gardoquia sericea C. Presl / Cordillera Peruvia / Haenke / Presl 1832». De acuerdo con esto, la colección MA 254984, pudiera considerarse material tipo (Isotypus) si no hay discordancia entre ambas plantas.

8. Glechon Spreng., consta de unas 7 especies que viven en el sur de Brasil, Paraguay, Uruguay y en el norte de Argentina (Harley in Harley et al. 2004: 245).

8.1. Glechon ciliata Benth., Lab. Gen. Sp. 408 (1834). Planta de América austral.

Glechon affinis Briq.; Glechon hassleri Briq.; Glechon squarrosa Benth. MA 254669, fl: [URUGUAY] «Bistropogon / 5 / ex monte Pan de azucar / in Montevideo / Neé iter» [et. ant.]; «Bistropogon. / 5 / Del monte Pan de azucar / de Montevideo [30-IX-1789] / Neé iter (Exp. Mal.)» [et. ant.]. Det. R. Morales, IV-2012.

9. Hedeoma Pers., consta de 42 especies que viven dispersas por toda América, desde Canadá hasta Argentina (Harley in Harley et al. 2004: 243).

9.1. Hedeoma multiflora Benth., Lab. Gen. Sp. 367 (1834). Planta del Cono Sur.

Hedeoma gilliesii Benth.; Micromeria bonariensis (Ten.) Fisch. \& C.A. Meyer; Satureja bonariensis (Fisch. \& C. A. Meyer) Briq. MA 257001, fr: «Thymus / Ex 47. / Neé iter» [et. ant.]; «Thymus / Neé iter (Exp. Mal.)» [et. tra.]. Det. R. Morales, XI-1990. 


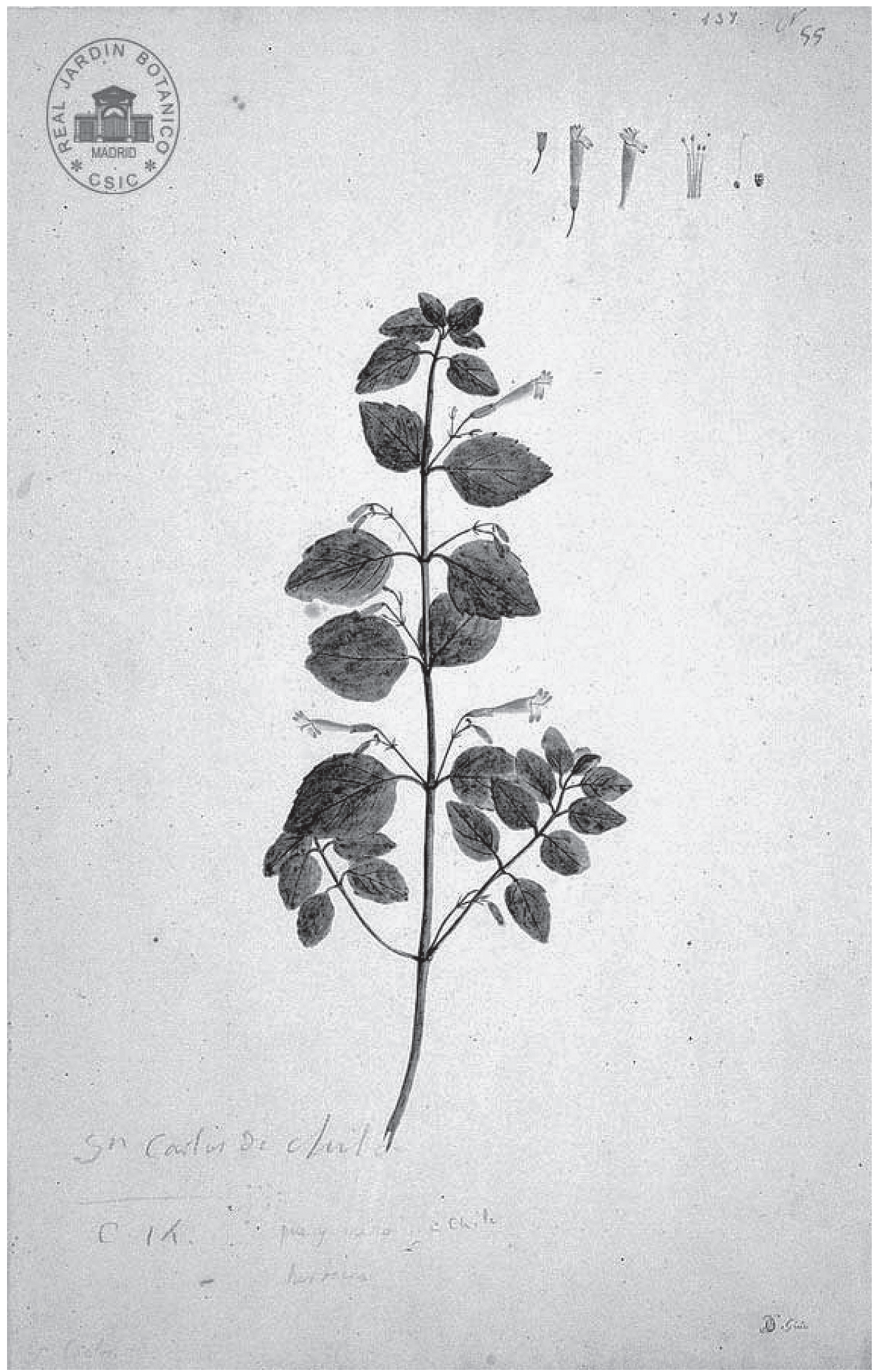

Fig. 1-Rizoa ovatifolia Cav. (Gardoquia multiflora Ruiz \& Pav.), Lamiaceae. José Guío. Aguada de colores sobre papel; 387 x 245 mm, Fondo Expedición Malaspina y José Bustamante (1789-1794). Luis Née y Tadeo Haenke. Archivo del Real Jardín Botánico de Madrid. Div. VI, 35. Sub Satureja multiflora (Ruiz \& Pav.) Briq. 

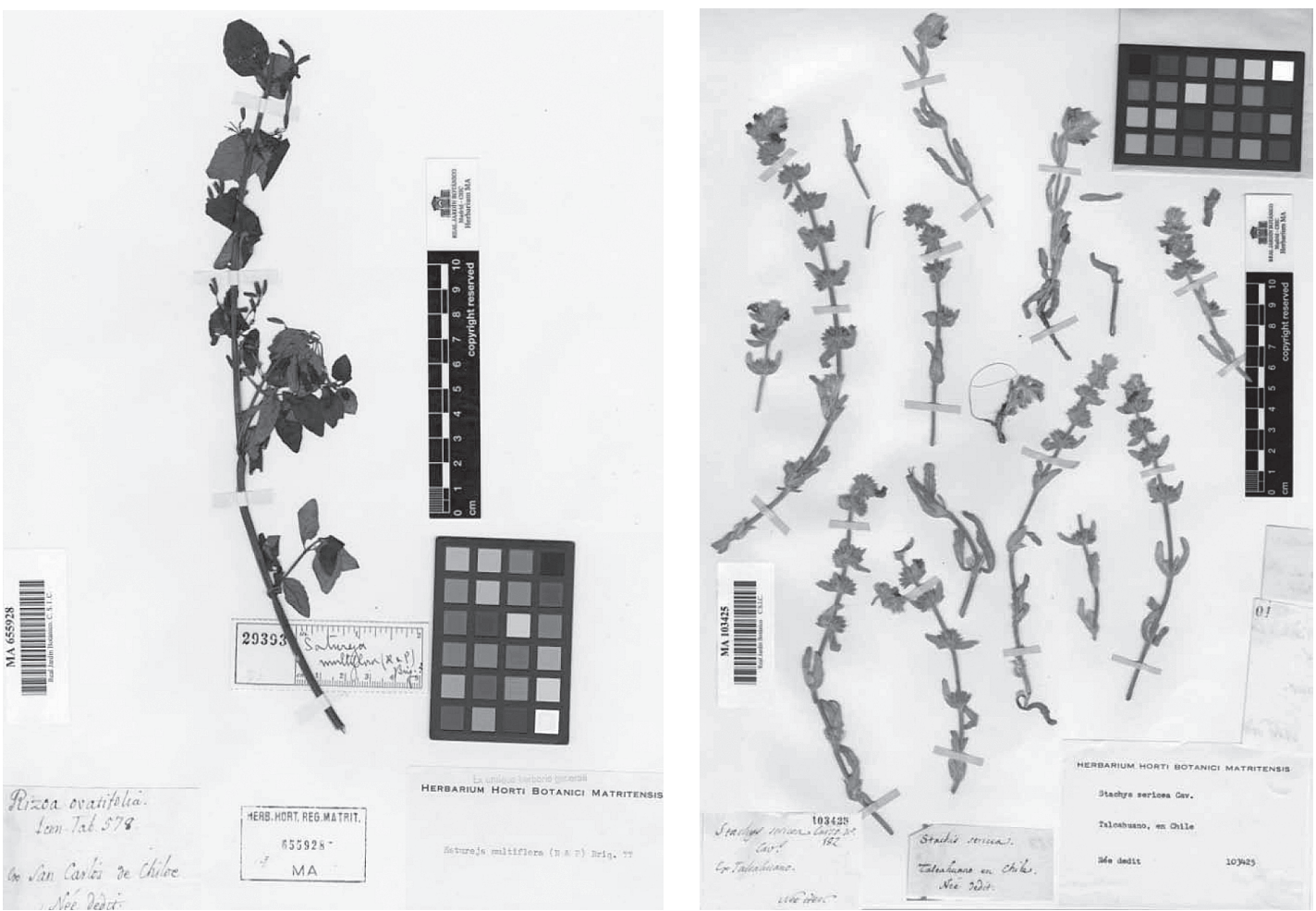

Fig. 2- A: Tipo de Rizoa ovatifolia Cav. (Gardoquia multiflora Ruiz \& Pav.), basado en una colección peruana de Neé. Pliego MA 655928, del herbario histórico de tipos de Cavanilles, en el Real Jardín Botánico de Madrid. B: Tipo de Stachys sericea Cav., basado en una colección chilena de Neé. Pliego MA 103425, del herbario histórico de tipos de Cavanilles.

9.2. Hedeoma piperita Benth., Lab. Gen. Sp.: 730 (1835). Planta de México.

MA 254594 fl: [MÉXICO] «Thymus? / 42 [II a XII-1791] / Neé iter» [et. ant.]; «Thymus? / Neé iter (Exp. Mal.)» [et. tra.]. Det. R. Morales, II-2012.

10. Hyptis Jacq., género casi exclusivamente americano con unas 290 especies, de las que muy pocas alcanzan zonas tropicales del Viejo Mundo. Crecen preferentemente en sabanas, cerrados y zonas abiertas. Hay varias espcies americanas introducidas por el hombre en otras zonas tropicales del mundo. (Harley in Harley et al. 2004, Fernández-Alonso 2010).

En este trabajo se sigue la circunscripción tradicional en este género (Epling 1949), sin incorporar las recientes segregaciones genéricas establecidas por Harley \& Pastore (2012), basadas en gran medida en análisis moleculares. Según esta nueva ordenación, de las 16 especies recolectadas en la expedición Malaspina aqui tratadas, 7 se mantendrían como Hyptis s. str., 4 pasarían al género Mesosphaerum Browne, 2 al género Cantinoa Harley \& Pastore, 3 a al gé- nero Condea Adanson y una al género Martianthus Harely $\&$ Pastore. De las 16 especies, la mayoría (8 sp.) son sudamericanas; otras se encuentran tanto en Sudamérica como en Mesoamérica ( 2 recolectadas en México, y 4 recolectadas en Panamá). Las especies recolectadas en Filipinas, son especies introducidas de origen antillano-mesoamericano.

10.1. Hyptis albida Kunth in Humb., Bonpl. \& Kunth., Nov. Gen. Sp. 2: 319 (1817)

Condea albida (Kunth) Harley \& Pastore; Mesosphaerum albidum (Kunth) Kuntze; MA 354626: [MÉXICO] «Inter Acapulco et Mexico [IV-XII-1791] / Neé dedit». Det. R. Harley, IV2011. MA 254666, fl: [MÉXICO] «Bistropogon? / Corola bilabiata; labium superius erec- / tum emarginatum; inferius tripartitum / lacinia intermedia amjori; filamenta pilosa / ex Peruvia / 8 / Neé iter» [et. ant.]; «Bistropogon / Perú / Neé iter (Exp. Mal.)» [et. tra.]. Det. R. Morales, XII-2012.

Esta especie vive en México y en el sur de Estados Unidos y se encuentra representada también en el herbario de Sessé y Mociño; no vive en Perú. Probablemente haya un error de etiquetado en el segundo espécimen. 
10.2. Hyptis atrorubens Poit., Ann. Mus. Natl. Hist. Nat. 7: 466, t 27, Fig. 3 (1806). Fig. 3A. Ampliamente distribuida en el neotrópico.

Hyptis tenella Briq. \& Spruce; Mesosphaerum atrorubens (Poit.) Kuntze; MA 255922-B, fl: [PANAMÁ?] «Salvia / 19 / ex. Neé iter» [et. ant.]; «Salvia / Neé iter (Exp. Mal.)» [et. tra.]. Det. J. L. Fern. Alonso, II-2013.

10.3. Hyptis elongata Benth., Lab. Gen. Sp.: 88 (1833). Planta del Perú.

Martianthus elongatus (Benth) Harley \& Pastore; Mesosphaerum elongatum (Benth.) Kuntze; MA 255057 fl, fr: [PERÚ] «Hyptis / de / Neé iter» [et. ant.]; «Hyptis / Neé iter (Exp. Mal.)» [et. tra.]. Det. R. Harley, X-2005. MA 255030, fl: [PERÚ] «¿Hyptis plumosa. / ex Perú [V a IX-1790] / Neé dedit» [et. ant.]; «¿Hyptis plumosa. / Cavanilles / Perú / Neé dedit (Exp. Mal.)» [et. tra.]. Det. R. Morales, III-2012.

El tipo de esta planta es una colección de Ruiz y Pavón procedente del Perú.

10.4. Hyptis floribunda Briq. ex Mich., Mem. Soc. Phys. Hist. Nat. Geneve 32(10): 18. 1897.

Mesosphaerum floribundum Briq.; Condea floribunda (Briq.) Harley \& Pastore, planta de Uruguay, Paraguay y Argentina. MA 754662: «[MÉXICO?/URUGUAY] Ex Nova Hispania / Nee iter [eti. antigua]; « Salvia; det: De Bredlone/ oct 1985; Det. J. L. Fern. Alonso, II-2013.

La localidad más probable de recolección de esta planta, considerando el recorrido de la expedición, es Montevideo. Esta es una de las localidades clásicas de esta planta restringia al Cono Sur. Sería este un caso más de cambio de etiquetas, con indicación errónea de México, donde con seguridad no vive esta especie (Epling 1949).

10.5. Hyptis florida Benth., Bot. Voy. Sulphur: 150 (1846). Planta de la región Pacífica de Colombia y Ecuador, poco conocida y muy escasa en los herbarios.

Mesosphaerum floridum Kuntze; MA 255053 fl, fr: [ECUADOR] «iHyptis / en el Real del monte, y en / Camino de Guayaquil al / Chimborazo. [4 a 21-X-1790] / Neé dedit.» [et. ant.]; «iHyptis / Real del monte y Camino de Guayaquil al / Chimborazo. / Cavanilles/ Neé dedit (Exp. Mal.)» [et. tra.]. Det. J. L. Fern. Alonso, 1990.

Aunque en las etiquetas originales de los dos pliegos de esta planta, se indican dos localidades diferentes, una de México «Real del Monte» y otra de Ecuador «Camino de Guayaquil al Chimborazo», la referencia a México es errónea, ya que se trata de una planta endémica de Ecuador-Colombia.
10.6. Hyptis lutescens Pohl ex Benth., Lab. Gen. Sp.: 109 (1833). Planta de Sudamérica.

Hyptis affinis Benth.; Mesosphaerum lutescens (Phol ex Benth.) Kuntze; MA 255070 (2 pliegos) fl, fr: [PERÚ] «Hyptis / ex Peruvia [V a IX-1790] / Neé iter» [et. ant.]; «Hyptis / Perú / Neé iter (Exp. Mal.)» [et. tra.]. Det. R. Harley, 10-X-2005.

10.7. Hyptis mutabilis (Rich.) Briq., Lab. Gen. Sp.: 109 (1833). Planta de Sudamérica.

Nepeta mutabilis Richard; Cantinoa mutabilis (Rich.) Harley \& Pastore; Hyptis polystachya Kunth. H. aspera Martins \& Galeotti; MA 255388A fr: «Nepeta// Guadalupe [México, etiqueta recortada] [V a IX-1791]» [et. ant., atribuible a Neé]; «Nepeta /Guadalupe / Cavanilles» [et. tra.]. Det. J. L. Fern. Alonso, II-2013.

10.8. Hyptis odorata Benth., Lab. Gen. Sp.: 81 (1833). Planta del Perú.

Mesosphaerum odoratum (Benth.) Kuntze; MA $754920 \mathrm{fl}$ : «Perú / Didynamia / Ex Huanuco (tachado) / Llama-/ pañaquin in Peruvia [V a IX-1790] / Neé dedit» [et. ant.]; «Verbenaceae, [et. tra.]». Det. J. L. Fern. Alonso, VI-2012.

En el herbario de Ruiz y Pavón en MA, también se encuentra una colección (MA 815018) de esta planta, con la anotación «Nepeta odorata Fl. Peruv. /Ex Herb. Fl. Peruv./ 1828».

10.9. Hyptis pectinata (L.) Poit., Ann. Mus. Natl. Hist. Nat. 7: 474, t 30 (1806). Fig. 3D. Ampliamente distribuida en el neotrópico.

Nepeta pectinata L.; Bystropogon pectinatus (L)L'Heritier; Mesosphaerum pectinatum (L.) Kuntze; Hyptis nepetoides Fisch. ex Schrank.; MA 255389, fr: [PERÚ] «Nepeta / an Bistropogon? / ex Peruvia / 11 [V a IX-1790] / Neé iter» [et. ant.]; «Nepeta an Bistropogon? / Perú / J.D. Rodríguez / Neé iter (Exp. Mal.)» [et. tra.]. Det. J. L. Fern. Alonso, I-2013. -MA 254916, fl, fr: [PERÚ] «Purruchuco / Perú» [et. ant.]; «Purruchuco / Perú [20 a 23 VII-1790] / Exp. Mal.» [et. tra. 1]; «Purruchuco / Perú» [et. tra. 1]. Det. J. L. Fern. Alonso, 1990.

10.10. Hyptis pilosa Benth., Lab. Gen. Sp.: 124 (1833). Planta de Sudamérica.

Mesosphaerum pilosum (Benth.) Kuntze; MA 254665, fl: [PERÚ] «Bistropogon / 16. / Neé iter peruvianum» [et. ant.]; «Bistropogon / Perú / Cavanilles / Neé iter (Exp. Mal. .) [et. tra.]. Det. R. Harley, 5-IV-2011. MA 254665, fl, fr: [CHILE] «Bistropogon / De Chile / 9. Neé iter» [et. ant.]; «Bistropogon / Chile / Neé iter (Exp. Mal.)» [et. tra.]. Det. R. Harley, 5-IV-2011.

10.11. Hyptis recurvata Poit., Ann. Mus. Natl. Hist. Nat. 7: 467, p 28, Fig. 2 (1806). Planta de América tropical.

Hyptis microcephala Bert. ex Benth.; Hyptis imitans Epling; Mesosphaerum recurvatum (Poit.) Kuntze; MA $255067 \mathrm{fl}$, fr: 
[PERÚ?] «Hyptis / Neé iter» [et. ant.]; «Hyptis / Neé iter (Exp. Mal.)» [et. tra.]. Det. R. Harley, 10-X-2005.

10.12. Hyptis savannarum Briq., Annuaire Conserv. Jard. Bot. Genève 2: 216 (1898). Fig. 3C. Planta de América tropical.

Mesosphaerum savannarum (Briq.) Kuntze; MA 255050 fl: [MÉXICO] «¿Hyptis / ex Nova-Hispania. [II a XII-1791] / Neé dedit» [et. ant.]; «iHyptis / Nueva-España / Cavanilles / Neé dedit (Exp. Mal.)» [et. tra.]. Det. R. Harley, IV-2011. MA 255063 fl, fr: [MÉXICO] «Iptis. / De N. España [II a XII-1791] / Neé iter» [et. ant. 1]; «Hyptis / de Panama / Neé iter» [et. ant. 2]; «Hyptis / Panama, Nueva España / Neé iter (Exp. Mal.)» [et. tra.]. Det. R. Harley, X-2005. MA 255065 (2 pliegos) fl, fr: [PANAMÁ] «Hyptis. / De Panama [17-XI a 10-XII-1790] / Neé iter» [et. ant.]; «Hyptis / Panama / Neé iter (Exp. Mal.)» [et. tra.]. Det. R. Harley, X-2005. MA 255068 fl, fr: [PANAMÁ] «Hyptis. / De Panamá [17-XI a 10-XII-1790] / Neé iter» [et. ant. 1]; «Hyptis / Panama / Neé iter (Exp. Mal.)» [et. tra.]. Det. R. Harley, X-2005.

10.13. Hyptis spicigera Lam., Encycl. 3: 185 (1789). Originaria de las Antillas, e introducida en otras zonas tropicales de América y del mundo.

Nepeta americana Aubl.; Hyptis americana (Aubl.) Urban non. Poir.; Mesosphaerum spicigerum (Lam.) Kuntze; Cantinoa americana (Aubl.) Harley \& Pastore; Hyptis subverticillata Anderson; H. gonocephala Wrigth ex Grisebach; MA $254655 \mathrm{fl}$, fr: [FILIPINAS] «Hissopus -tachado- / Bistropogon / Ex insulis Philippicis [III a XII-1792] / Neé iter» [et. ant.]; «Bystropogon. / Islas Filipinas / Neé iter (Exp. Mal.)» [et. tra.]. Det. J. L. Fern. Alonso, II-2013. MA 254670 fl: [MEJICO] «Bistropogon 15/ Ex Nova Hispania / Neé iter» [et. ant.]; «Bystropogon. / Nueva España / Neé iter (Exp. Mal.)» [et. tra.]. Det. J. L. Fern. Alonso, II-2013. MA $255391 \mathrm{fl}$, fr: [FILIPINAS] «Nepeta? / Ex insulis Philippicis [III a XII-1792] / Neé dedit» [et. ant.]; «Nepeta? / Islas Filipinas / Cavanilles Neé dedit (Exp. Mal.)» [et. tra.]. Det. J. L. Fern. Alonso \& R. Morales, II-2013.

Existen registros posteriores de Filipinas (Keng, 1978; Pelser et al., 2011), aunque los que aquí se indican son sin duda las primeras colecciones de esta especie documentadas en aquella región.

10.14. Hyptis suaveolens (L.) Poit., Ann. Mus. Natl Hist. Nat. 7: 472, t 29, Fig. 2 (1806). Figura 3B. Planta de América tropical, naturalizada en otras regiones tropicales del mundo.

Ballota suaveolens L., Syst. Nat., ed. 10, 2: 1100 (1759); Mesosphaerum suaveolens (L.) Kuntze; Hyptis plumieri Poiteau; Hyptis congesta Leonard; MA 254653 fl, fr: [MÉXICO] «Bistropogon / St. Thomas / (173)» [et. ant. 1]; «ex nova-hispania / Neé dedit» [et. ant. 2]; Bystropogon suaveolens? / L'heritier Sert.
Ang. pag. 19/ ex insula S. Thomae. / LeDru dedit.» [et. ant. 1]; «Bystropogon suaveolens? / L'heritier Isla de Santo Thomas. / Cavanilles / LeDru dedit.» [et. tra.]. Det. R. Morales, III-2012. En este pliego hay ejemplares de dos localidades diferentes. MA 254660: [MÉXICO] «Bistropogon / 12. / e//x Guanajuato [19 a 23-X-1790] / Neé iter». MA 254663 fl: [MÉXICO] «Bistropogon / 18. / Ex Acapulco [8-V-1791] / Neé dedit» [et. ant.]; «Bistropogon / Acapulco / Neé iter (Exp. Mal.)» [et. tra.]. Det. R. Morales, IV-2012. MA 254654 fl: [FILIPINAS]«Bistropogon / 19. / Insul. Philipp. et Marian. [II a XII-1792] / Neé dedit» [et. ant.]; «Bistropogon / Islas Filipinas y Marianas / Cavanilles / Neé dedit» [et. tra.]. Det. R. Morales, IV-2012. MA 255249 fl, fr: [PANAMÁ] «Melissa / ex Panamaide [17-XI a 10-XII-1790] / Neé iter» [et. ant.]; «Melissa / Panama / Neé iter (Exp. Mal.)» [et. ant.]. Det. R. Harley, X-2005. MA 255250: fl, fr: [MÉXICO] «Melissa / ex nov. Hispania / Neé iter» [et. ant.]; «Melissa?/ Nueva España / J. D. Rodríguez / Neé iter (Exp. Mal.)» [et. tra.]. Det. R. Harley, III-1996.

10.15. Hyptis uncinata Benth., Lab. Gen. Sp.: 80 (1833). Planta de Sudamérica.

Mesosphaerum uncinatum (Benth.) Kuntze; MA $255064 \mathrm{fl}$, fr,: [PERÚ] «Hyptis? / del Perú [V a IX-1790] / Neé iter» [et. ant.]; «Hyptis? / Perú / Neé iter (Exp. Mal.)» [et. tra.]. Det. R. Harley, X-2005.

10.16. Hyptis verticillata Jacq., Icon. Pl. Rar.: 101 (1787). Planta ampliamente distribuida en el neotrópico.

Mesosphaerum verticillatum (Jacq.) Kuntze; Condea verticillata (Jacq.) Harley \& Pastore; Mentha hyptiformis Poiret; Hyptis parviflora Martens \& Galeotti; MA 255300, fl: [PANAMÂ] «Mentha? / ex Panamaide [17-XI a 10-XII-1790] / Neé iter» [et. ant.]; «Mentha? / Panamá / J. D. Rodriguez / Nee iter (Exp. Mal.)» [et. tra.]. Det. R. Morales \& J. L. Fern. Alonso, XII-2012

11. Lepechinia Willd., consta de unas 40 especies que viven desde California, a traves de Mesoamérica y de los Andes hasta Chile (Harley in Harley et al. 2004: 236). Una especie de México y las otras 5 de Sudamérica.

11.1. Lepechinia caulescens (Ortega) Epling, Repert. Spec. Nov. Regni Veg. Beih. 85: 20 (1935). Planta de México.

Horminum caulescens Ortega, Nov. Rar. Pl. Descr. Dec.: 63 (1798). MA 255000, fl: [MÉXICO] «Horminum caulescens / Ortega. Decad. / ex Nova Hispania [III a XII-1791] / Neé iter» [et. ant.]; «Horminum caulescens Ortega. Decad. Nueva / J. D. Rodríguez / Neé iter (E. M.)» [et. tra.]. Det. R. Morales, VI1991. MA 255001, fl: [MÉXICO] «Horminum caulescens / Ortega. Decad. P. 63. T. 8 / ex R. H. Mat. 1798» [et. ant.]; «Horminum caulescens Ortega. / Hort. Reg. Matr. / Cavanilles» [et. tra.]. Det. R. Morales, VI-1991. 

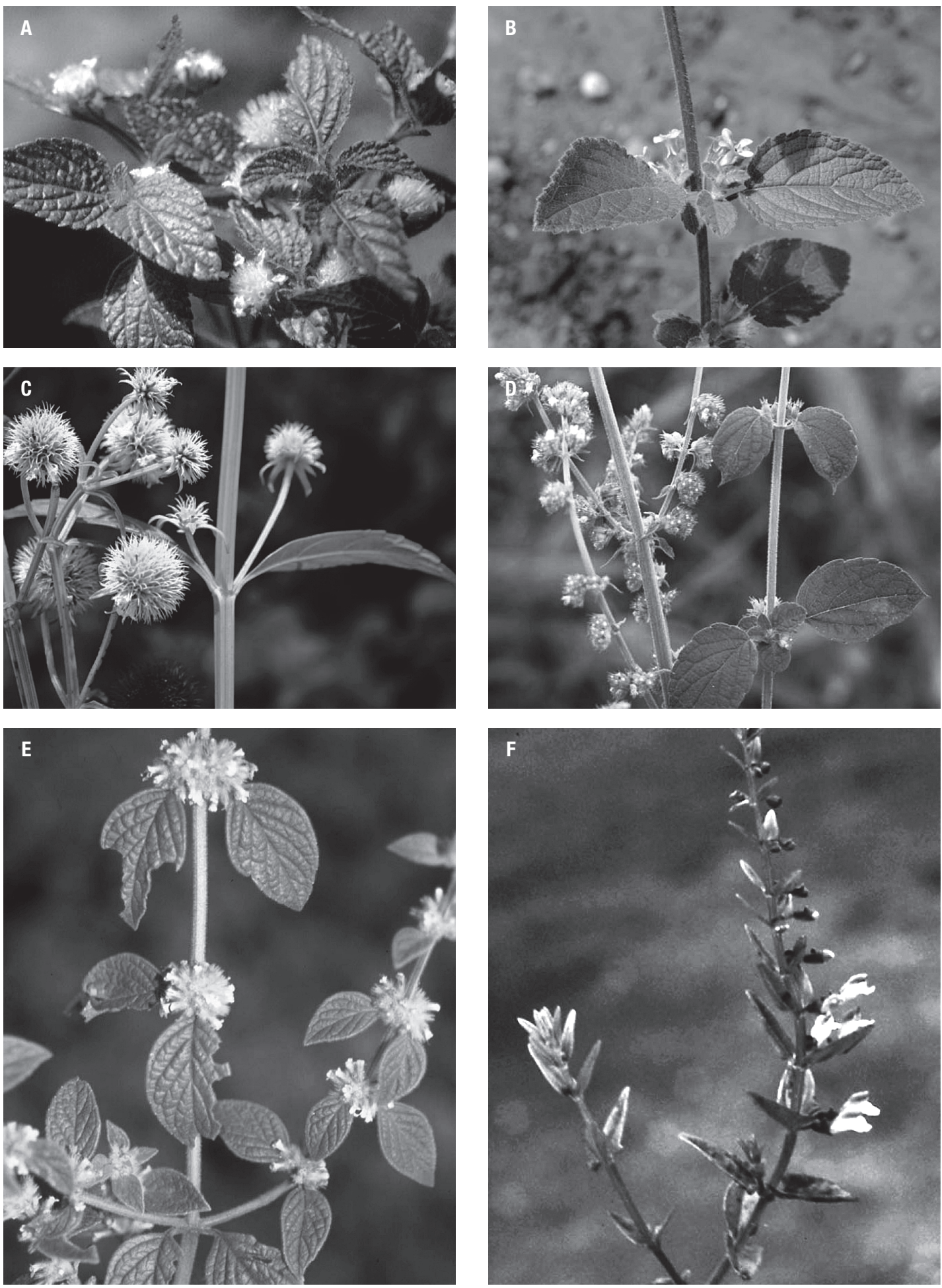

Fig. 3- Imágenes de alguna de las especies americanas tratadas. A: Hyptis atrorubens Poit. B: Hyptis suaveolens (L.) Poit. C: Hyptis savannarum Briq. D: Hyptis pectinata (L.) Poit. E: Minthostachys mollis (Kunth) Griseb. F: Scutellaria racemosa Pers. (Fotografias J. L. Fernández Alonso). 
Aunque en la etiqueta de la segunda colección (MA 2550019), no aparece la indicación de Neé, se trata sin duda de una planta procedente también de esta expedición.

11.2. Lepechinia chamaedryoides (Balb.) Epling, Repert. Spec. Nov. Regni Veg., Beih. 85: 20 (1935). Planta de América austral.

Dracocephalum chamaedryoides Balb. Misc. Bot.: 29 (1804); Sphacele chamaedryoides (Balb.) Briq.; Sphacele campanulata Benth.; MA 255006-1 (2 pliegos), fl, fr: [CHILE] «La didynamia / Romero vulgo / frutex habitat chile / flores obri / Horminium?» [et. ant.]; «Horminium? / (vulgo Romero) Chile / Florece abril» [et. ant.]. Det. R. Morales \& J. L. Fern. Alonso, 2012. MA 255006-2, fl, fr: [CHILE] «Horminium? / (vulgo Romero) Chile / Florece abril /» [et. tra.]. Det. R. Morales \& J. L. Fern. Alonso, 2012. MA 255857, fl: [PERÚ] «Salvia / 49 / ex Perú [V a IX-1790] / Neé dedit» [et. ant.]; «Salvia / Perú / Cavanilles / Neé dedit (Exp. Mal.)» [et. tra.]. Det. J. L. Fern. Alonso, VII-2012. MA 255860 (2 pliegos) fl, fr: [CHILE] «Salvia / ex Talcahuano [XI-1793] / Neé iter» [et. ant. 1]; «Salvia / 50 / del Perú y Chile / Neé iter» [et. ant. 2]; «Salvia. / Talcahuano in Chile / Neé dedit» [et. ant. 3]; «Talcahuano / Salvia.» [et. ant. 4]; «Salvia / Talcahuano en Chile y Perú / Neé iter (Exp. Malapina).» [et. tra.]. Det. R. Morales \& J. L. Fern. Alonso, III-2012. MA 255864, fr: [CHILE] «Salvia? / Romero vulgo / Chile julio /51» [et. ant., atribuible a Neé]; « «Salvia? / (vulgo Romero) / Chile» [et. tra.]. Det. J. L. Fern. Alonso, VII-2012. MA 255879, fl: [CHILE] «Salvia 102 / Talcahuano en Chile [XI-1793] / Neé dedit» [et. ant.]; «Salvia / Talcahuano en Chile / Cavanilles / Neé $\operatorname{dedit~(E.M.)»~[et.~tra.].~Det.~R.~Morales~\& ~J.~L.~Fern.~Alonso.~-~}$ MA 256902, esteril: [CHILE] «Teucrium / de Talcahuano [XI1793] / Neé iter» [et. ant.]; «Teucrium / Talcahuano/ Neé iter (Exp. Mal.)» [et. tra.]. Det. J. L. Fern. Alonso, II-2013. MA 256913, fl,fr: [CHILE] «Teucrium? / Neé iter» [et. ant.]; «Teucrium? / J. D. Rodríguez / Neé iter (Exp. Mal.)» [et. tra.]. Det. J. L. Fern. Alonso, II-2013. MA 292416, esteril: [CHILE] «Salvia? / año 1804 / Neé dedit» [et. tra.]. Det. J. L. Fern. Alonso, II-2013.

11.3. Lepechinia lamiifolia (Benth.) Epling, Repert. Sp. Nov. Regni Veg. Beih. 85: 21 (1935). Planta de Perú.

Sphacele lamiifolia Benth., Lab. Gen. Spec.: 570 (1834). MA 255858(1), fr: [PERÚ]»Salvia / Perú iuxta Obragillo. [18 a 28-VI-1790] / Neé dedit» [et. ant.]. Det. J. L. Fern. Alonso \& R. Morales, 2012. MA 255858(2), fr: [PERÚ] «Salvia / Del Perú [V a IX-1790] / Neé iter» [et. ant.]; «Salvia / The same plant from Huasa-huasi. / Perú / Neé» [et. tra.]. Det. R. Morales \& J. L. Fern. Alonso, 2012.

11.4. Lepechinia sagittata Epling, Bull. Torrey Bot. Club 71: 487 (1944). Planta de América austral.

MA $255862 \mathrm{fl}$, fr: [ARGENTINA] «Salvia? / Neé iter» [et. ant. 1]; «Salvia? / Cerro de las Pampas de / Buenos-Ayres. / Neé dedit» [et. ant. 2]; «Salvia? / Cerro de las Pampas de / Buenos-
Aires. / Neé dedit (E.M.)» [et. tra.]. Det. R. Morales \& J. L. Fern. Alonso, 1991.

«Lepechinia salviifolia» (Kunth) Epling, Repert. Spec. Nov. Regni Veg. Beih. 85: 23 (1935).

MA 255005: [COLOMBIA] «Horminium/ ex Peruvia/Neé iter» [et. ant.]; «Horminium / Perú / J.D. Rodríguez / Neé iter (Exp. Mal.)» [et. tra.]. Det. J. L. Fern. Alonso, II-2012.

Esta especie es endémica de Colombia y no se encuentra en Perú (Fernández Alonso 2002). El ejemplar proviene de la colección Mutis, de alguno de los envíos de muestras efectuados por Mutis a Madrid (1773-1808).

11.5. Lepechinia subhastata (Benth.) Epling, Repert. Spec. Nov. Regni Veg. Beih. 85: 22 (1935). Planta de Perú y Chile.

Sphacele subhastata Benth., Edwards's Bot. Reg. 15: sub t. 1289 (1829). MA 255872 fr: [CHILE] «Salvia / 12 / Chile / Neé dedit» [et. ant.]; «Salvia / Chile / Cavanilles / Neé dedit (E.M.).» [et. tra.]. Det. R. Morales \& J. L. Fern. Alonso, VI1991. MA 255919 fl: [PERÚ] «Salvia / 13 / Del Perú [V a IX1790] / Neé iter» [et. ant.]; «Salvia / Perú / Neé iter (E.M.).» [et. tra.]. Det. R. Morales \& J. L. Fern. Alonso, VI-1991.

11.6. Lepechinia vesiculosa (Benth.) Epling, Repert Sp. Nov. Regni Veg. Beih. 85: 24 (1935). Planta de Argentina.

Hyptis vesiculosa Benth., Labiat. Gen. Spec. 131 (1833); Alguelagum auriferum Rusby; Astemon graveolens Regel; Sphacele inflata Briq.; Lepechinia inflata (Briq.) Epling; Sphacele kuntzeana Briq.; Sphacele vesiculosa (Benth.) J. F. Macbr.; MA 254944, fl, fr: [ARGENTINA] «Dracocephalum» [coleccion atribuible a Neé]. Det. J. L. Fern. Alonso, II-2012. MA 257207, fl, fr: [ARGENTINA] «Leonurus / Neé iter?. Det. J. L. Fern. Alonso, II-2012.

12. Leucas R. Br., género con unas 100 especies que se extienden desde África tropical y del sur hasta el SE de Asia y Japón (Ryding in Harley et al. 2004: 227).

12.1. Leucas zeylanica (L.) R. Br., Prodr. Fl. Nov. Holland. 504 (1810). Planta del SE de Asia.

Phlomis zeylanica L., Sp. Pl. 2: 586 (1753). MA 255186 fl: [FILIPINAS] «Leonurus / de / Filipinas [III a XII-1792] / Neé iter « [et. ant. 1]; «Stachis / Me... / en los fosos de / ....» [et. ant. 2]; « Leonurus / Islas Filipinas / Neé iter « [et. tra.]. Det. R. Morales, X-1998.

12.2. Leucas lavandulifolia Sm. in Rees, Cycl. 20. 1812. Planta de Asia y Oceanía.

Leonurus indicus L.; Phomis linifolia Roth.; MA 255185 fl, fr: [FILIPINAS] «Leonurus / ex Philippicis insulis [III a XII- 
1792] / Neé iter» [et. ant.]; «Leonurus / Islas Filipinas / J.D. Rodríguez / Neé iter (Exp. Mal.)» [et. tra.]. Det. R. Morales, X1998. MA 255183 fl, fr: [FILIPINAS] «Leonurus / ex Filipinas [III a XII-1792] / Neé iter» [et. ant.]; «Leonurus / Islas Filipinas / Neé iter (Exp. Mal.)» [et. tra.]. Det. R. Morales, X-1998.

12.3. Leucas marrubioides Desf., Mem. Mus. Hist. Nat. 11: 6, t 3 Fig. 1 (1824). Planta de Asia.

MA 255182 fl, fr: [FILIPINAS] «Leonurus / Del Perú / Neé iter» [et. ant.]; «Leonurus / Perú / Neé iter (Exp. Mal.)» [et. tra.]. Det. R. Morales \& J. L. Fern. Alonso, II-2013.

\section{Minthostachys Griseb.}

Consta de unas 10 especies que viven en América, en los países andinos desde Venezuela hasta Argentina (Harley in Harley et al. 2004: 241). Según Schmidt-Lebuhn (2008), son 17 las especies de Mintostachys.

13.1. Minthostachys mollis (Kunth) Griseb., Abh. Königl. Ges. Wiss. Göttingen 19: 235 (1874). Fig. 3E. Planta de Sudamérica.

Bystropogon mollis Kunth, in Humb., Bonpl. \& Kunth, Nov. Gen. Sp. Pl. 2: 317 (1816); Mentha mollis (Kunth) Benth., Lab. Gen. Sp.: 182 (1832). MA 255291 fl, fr: [PERU'] «Mentha / del Perú [V a IX-1790] / Neé iter» [et. ant.]; «Mentha / Perú / Neé iter (E.M.)» [et. tra.]. Det. R. Morales, VIII-2002. MA 255298 fl, fr (2 pliegos): [PERÚ] Mentha? / ex Panamaide et Peruvia / Neé iter» [et. ant.]; «Mentha / Panamá y Perú / J.D. Rodríguez / Neé iter (E.M.)» [et. tra.]. Det. R. Morales, VIII-2002. MA 255301 fl, fr: [PERU'] «Mentha / Neé iter» [et. ant.]; «Mentha / Neé iter (E.M.?)» [et. tra.]. Det. R. Morales, VIII-2002.

La indicación de su presencia en Panamá es incorrecta.

14. Ocimum L., consta de unas 65 especies que viven en los trópicos de todo el mundo (Paton in Harley et al. 2004: 260).

14.1. Ocimum americanum L., Cent. Pl. 1: 15 (1755). Planta aparentemente originaria del Viejo Mundo.

Ocimum canum Sims; Ocimum stramineum Sims; Ocimum fruticulosum Burch.; MA 255426, fl, fr: [FILIPINAS] «Ocymum / Ex insulis Philippicis [III a XII-1792] / Neé dedit» [et. ant.]; «Ocymum / Islas Filipinas / Cavanilles / Neé dedit (Exp. Mal.)» [et. tra.]. Det. J. L. Fern. Alonso, I-2013. MA 256224, fl, fr: [FILIPINAS] «Scutellaria? / Camarines in insula Luzon [IV a VI-1792] / Neé dedit» [et. ant.]; «Scutellaria? / Camarines en Isla de Luzón / Cavanilles / Neé dedit» [et. tra.]. Det. J. L. Fern. Alonso, I-2013.

15. Origanum L., consta de unas 40 especies que viven sobre todo en la región circunmediterránea, aunque una de ellas, Origanum vulgare, se extiende por el continente asiático. (Harley in Harley et al. 2004: 238). Varias de sus especies se cultivan desde antiguo, sobre todo el orégano (O. vulgare) y la mejorana (O. majorana).

15.1. Origanum x majoricum Cambess., Mém. Mus. Hist. Nat. 14: 296 (1827) pro sp.

Origanum x applii Boros; MA 255450, fl: [PERÚ] «Origanum / 28 / ex oppido Puruchuco 18 / leucis a Lima [VII-1790]. / Neé dedit» [et. ant.]; «Origanum x majoricum Cambess. Det. R. Morales / De Puruchuco en Lima/ Cavanilles/ Neé dedit (Exp. Mal.)» [et. tra.].

16. Plectranthus L'Hérit., incl. Solenostemon Thonn y Coleus Lour. Género que consta de unas 300 especies que viven en los trópicos del Viejo Mundo (Paton in Harley et al. 2004: 265).

16.1. Plectranthus parviflorus Willd., Enum. Hort. Berol. 1: t 65 (1806). Planta de Asia y Australia.

MA 255423A, fr: [AUSTRALIA] «Ocimum / Bahia botanica [III a IV-1793] / Neé dedit» [et. ant.]; «Ocymum / Bahia Botanica / Cavanilles 7 Neé dedit (Exp. Mal.)» [et. tra.]. Det. R. Morales/ II-2013. (pro parte). MA 255425 (dos pliegos), fl, fr: [AUSTRALIA] «Ocymum / de Jackson en B. Botan. [III a IV1793] / Neé iter» [et. ant.]; «Ocymum / Jackson en Bahia Botanico / Neé iter (Exp. Mal.)» [et. tra.]. Det. R. Morales, II-2013.

16.2. Plectranthus rotundifolius (Poir.) Spreng., Syst. Veg., ed. 16, 2: 690 (1825). Planta del Paleotrópico, que se cultiva por sus rizomas.

Germanea rotundifolia Poiret in Lam., Encycl. 2: 763 (1812). MA 255429, fl: [FILIPINAS] «Ocymum? / Neé iter» [et. ant.]; «Ocymum? / Neé iter (E.M.)» [et. tra.]. Det. R. Morales, I-2012. MA 256900, fl: [FILIPINAS] «Teucrium / inter Mauban et / Caventi. Luzón [6 a 13-VI-1792] / Neé dedit» [et. ant. 1]; Mayana; pinag / Libingan /» [et. ant. 2]; «Teucrium? / Entre Mayan y Cavente Luzon / Neé dedit» [et. tra.]. Det. R. Morales, I-2012.

17. Pogostemon Desf., consta de unas 80 especies que viven sobre todo en el sur y este de Asia, algunas en África tropical y Australia, en bosques o lugares encharcados (Ryding in Harley et al. 2004: 214).

17.1. Pogostemon auricularius (L.) Hassk., Tijd. Nat. Gesch. Phys. 10: 127 (1843). Planta de Filipinas.

Mentha auricularia L., Mant. P1.: 81 (1767); Dysophylla auricularia (L.) Blume; Mentha foetida Burm. f.; MA 257238 , fl: [FILIPINAS] «Mentha / ex Sambuangan [23-XI a 6-XII1792] / Neé iter» [et. ant. 1]; Samboaga [et. ant. 2]; «Mentha / 
de Sambuangan [23-XI a 6-XII-1792] / Neé iter (E.M.)» [et. tra.]. Det. R. Morales, XII-2012. MA 292452, fl: [FILIPINAS] «Islas filipinas y Marianas [II a XII-1792] / Neé iter» [et. ant.]. Det. R. Morales \& J. L. Fern. Alonso, II-2013.

18. Poliomintha A. Gray, consta de unas 7 especies que viven en el suroeste de Estados Unidos y norte de México; también en Haití (Harley in Harley et al. 2004: 244).

18.1. Poliomintha marifolia (S. Schauer) A. Gray, Proc. Amer. Acad. Arts 8: 365 (1872). Planta de México.

Keithia marifolia S. Schauer, Linnaea 20: 705 (1847); Hedeoma marifolia (F. Schauer) Briq.; Hesperozygis marifolia (S. Schauer) Epling, MA 255882 fl, fr: [MÉXICO] «Salvia । 68 / De N. España [II a XII-1791] / Neé iter» [et. ant.]; « «alvia / Nueva España / Neé iter (E.M.)» [et. tra.]. Det. S. Zamudio, IV-2012.

19. Prunella L., consta de 7 especies que viven en Eurasia y el noroeste de África, aunque algunas de ellas se han naturalizado por el resto del mundo (Morales in Harley et al. 2004: 260).

19.1 Prunella vulgaris L., Sp. Pl. 2: 600 (1753). Planta introducida de Europa.

Prunella aequinoctialis Kunth, MA 255882, fl, fr: [AUSTRALIA] «Prunella / ex nov. Hollandia [III a IV-1793] / Neé iter» [et. ant.]; «Prunella / Nueva Holanda / J.D. Rodríguez / Neé iter (Exp. Mal.)» [et. tra.]. Det. R. Morales \& J. L. Fern. Alonso, II-2013.

20. Salvia L., es el género de labiadas mejor representado en la colección de la expedición Malaspina (164 pliegos/46 spp.), por lo que será motivo de otra publicación, segunda parte de este catálogo (Fernández Alonso, en preparación). La mayoría de las salvias recolectadas por Neé procede de territorio mejicano (30 especies), y el resto (16 especies), proviene de los Andes.

21. Scutellaria L., género con unas 360 especies que viven practicamente en todo el mundo (Cantino in Harley et al. 2004: 211), a excepción de algunas regiones áridas donde es muy escaso 0 está ausente.

21.1. Scutellaria dumetorum Schltdl., Linnaea 7: 400 (1832). Planta de Norteamérica.

incl. S. coerulea Moc. \& Sessé ex Benth.; MA 254942, fl: [MÉXICO] «Dracocephalum / an Scutellaria? / ex. nov. Hispania [II a XII-1791] / Neé iter» [et. ant.]; «k / an Scutellaria? / Nueva España / Neé iter (Exp. Mal.)» [et. tra.]. Det. J. L. Fern. Alonso, II-2013. MA 255922A, fl: [MEXICO] «Salvia / 19/ ex.
Neé iter» [et. ant.]; «Salvia / Neé iter (Exp. Mal..)» [et. tra.]. Det. J. L. Fern. Alonso, II-2013. MA 256223, fl: [MÉXICO] «Scutellaria / 46 / De Chalma [18-VII a 24-VIII-1791] / Neé iter» [et. ant.]; « «Scutellaria / Talcahuano / Neé iter (Exp. Mal.)» [et. tra.]. Det. J. L. Fern. Alonso, II-2013. -MA 256274A, fl: [MÉXICO] «Stachis / de Nueva España [II a XII-1791] / Neé iter» [et. ant. 1]; « «Stachys / Nueva España / Neé iter (Exp. Mal.)» [et. tra.]. Det. J. L. Fern. Alonso, XII-2012; B pro parte.

21.2. Scutellaria ocymoides (Kunth) Epling, Repert. Sp. Nov. Beih. 85: 178 (1936). Planta de Sudamérica.

Perilomia ocymoides Kunth in Humb., Bonpl. \& Kunth, Nov. Gen. Sp. 2: 328 (1818); MA 255457/1, fl, fr: [PERÚ] «SCutellaria / Perú [V a IX-1790] / Neé dedit» [et. ant. 1]; «Scutellaria / ex Nov / Neé iter» [et. ant. 2]; «Ex. Ant. Herb. Generali / Perilomia ocymoides Kunth» [et. tra.]. Det. R. Morales, III-2012. MA 255457/2, fr: [CHILE] «Scutelaria / De Chile / Neé iter» [et. ant.]; «Ex. Ant. Herb. Generali/ Perilomia ocymoides Kunth» [et. tra.]. Det. R. Morales, III-2012. Hay dos duplicados más, ambos con flor y fruto. MA 255894: [PERÚ] «Salvia / N. E. Actopan» [mezclada con Salvia]. MA 256225, fl: [PERÚ] «1. Iuxta Guanamantanga in / Perú. [6 a 18-VII-1790] / Neé dedit» [et. ant. 1]; «Scutellaria? / An Ocymum Sp. / Guanamantanga in Regni / Perú» [et. ant. 2]. Det. J. L. Fern. Alonso \& R. Morales, II-2013.

21.3. Scutellaria racemosa Pers., Syn. P1. 2(1): 136 (1806). Fig. 3F. Planta del cono sur.

S. rumicifolia Kunth; S. bonariensis Willd. ex Benth.; S. hastata Larrañaga; S. heterophylla Willd. ex Benth.; MA 256218, fl: [CHILE] «Scutellaria? / De Jalcahuano [XI-1793] / Neé iter» [et. ant.]; «Scutellaria? / Talcahuano / Neé iter (Exp. Mal.)» [et. tra.]. Det. J. L. Fern. Alonso, 1990. MA 256219, fl: [URUGUAY] «Scutellaria / Ex Montevideo [IX a X-1789] / Neé iter» [et. ant.]; «Scutellaria / Montevideo / Cavanilles / Neé iter (Exp. Mal.)» [et. tra.]. Det. J. L. Fern. Alonso, 1990.

21.4. Scutellaria sp. Planta de México. En el pliego MA 255894 aparecen montadas dos plantas diferentes, que han sido numeradas como A y B. En el pliego MA 255894A, fl, aparecen tres ramillas de Priva aspera Kunth (Verbenaceae).

MA 255894B, fr (pro parte): [MÉXICO] «Salvia / 44. / De N. España [III a XII-1791] / Neé iter» [et. ant. 1]; «N. E. Actopan / Salvia» [et. ant. 2]; «Salvia / Nueva España / Neé iter (Exp. Mal.)» [et. tra.]. Det. J. L. Fern. Alonso \& R. Morales, 1990.

22. Stachys L., género subcosmopolita con unas 300 especies. Su centros de distribución se encuentran en el Mediterráneo, SO de Asia, América y Sudáfrica (Krestovskaja in Harley et al. 2004: 223). De las 8 especies identificadas en el herbario de la expedición Malaspi- 
na, cuatro provienen de México y de Chile y una de $\mathrm{Ca}$ nadá.

22.1. Stachys bridgesii Benth., Lab. Gen. Sp.: 546 (1834). Planta de Chile.

MA 256681, fl: [CHILE] «Stachys / Neé iter» [et. ant.]; «Stachys / Neé iter (Exp. Mal.)?» [et. tra.]. Det. J. L. Fern. Alonso, II-2013. MA 256684, fl, fr: [CHILE] «Stachys / Del Planchon / Neé iter» [et. ant.]; «Stachys / Del Planchon / Neé iter (Exp. Mal.)?» [et. tra.]. Det. J. L. Fern. Alonso, II-2013. MA 256688, fl, fr: [CHILE] «Stachys / Ex peruvia et Chile / Neé iter» [et. ant.]; «Stachys / Perú y Chile / Neé iter (Exp. Mal.)?» [et. tra.]. Det. J. L. Fern. Alonso, II-2013.

22.2. Stachys calcicola Benth., Bull. Torrey Bot. Club 71: 484 (1944). Planta de México.

MA 256680, fl: [MÉXICO] «Stachys / ex nov. Hispania [III a XII-1791] / Neé iter» [et. ant.]; «Stachys / Nueva españa / J. D. Rodríguez / Neé iter (Exp. Mal..)? [et. tra.]. Det. J. L. Fern. Alonso, II-2013.

22.3. Stachys coccinea Ortega, Nov. Pl. Descr. Dec.: 20 (1797). Planta de México.

S.coccinea Jacq., Pl. Hort. Schoenb. 3: 18, pl. 284. 1798. [Cav., Icon 6: 37 (1801)]: «Habitat in Chalmae ditione, Novae Hispaniae oppidi, ibique ... Stachys coccinea ...». Basado en una colección mejicana de Chalma (Muñoz Garmendia 1992: 384). MA 231455, fl: [MÉXICO] «Neé / Stachis cocinea / Ortega» [et. ant. 1]; «Stachys cocinea Ortega / ex nov. Hispania [III a XII-1791] / Neé iter» [et. ant. 2]; «B. B. Munro 1983 / Not type material of: Stachys coccinea Ortega... / this collection appears to have come from Neé from Mexico...» [et. tra.]. MA 231456 fl, fr: [MÉXICO] «Stachis cocinea Ortega decad. psg 20 / ex R. h. Mat. Aug. 1797/ ex nova hispania 7» [III a XII-1791] [et. ant. 1]; «en los pinares de Ocuila cerca de Chalma [18-VII a 24-VIII1791] / N. Esp. / Neé dedit» [et. ant. 2]; «B. B. Munro 1983 / Not type material of: Stachys coccinea Ortega.../ This specimen was collected by Neé in Mexico then groun in Reg. Hort. Matri. / The type description says Sessé supplied the plant.» [et. tra.]. MA 231457 fl, fr: «Stachys coccinea Ortega Dec. ex Hort. Reg. Matrit. anno 1800» [et. ant.]; «Ex antiquo Herb. Gener. / Hort. Reg. Bot. Matr.» [et. tra. 1]; «0. B. Munro, 1983 / Possible Isotype of Stachys coccinea Ortega, Hort. Matr. 20. 1797. This collection came from material in the Hort. Reg. Bot. Matr. in Matrit and may have been from the type plant wich is perennial» [et. tra. 2]. MA 256274A, fl: [MÉXICO] «Stachis / de Nueva España [III a XII-1791] / Neé iter» [et. ant. 1]; «Stachys / Nueva España / Neé iter (Exp. Mal.)» [et. tra.]. A, pro parte. Det. J. L. Fern. Alonso, XII-2012. MA 256275, fl, fr: [MÉXICO] «Stachys / ex nov. Hispania [III a XII-1791] / Neé iter» [et. ant.]; «Stachys / Nueva España / J. D. Rodríguez / Neé iter (Exp. Mal.)» [et. tra.]. Det. J. L. Fern. Alonso, XII-2012. MA 256687, fl, fr: [MÉXICO] «Stachys / Neé iter» [et. ant.]; «Stachys / J.D. Ro- dríguez / Neé iter (Exp. Mal.?)» [et. tra.]. Det. J. L. Fern. Alonso, XII-2012.

22.4. Stachys emersonii Piper, Erythea 6: 31 (1898). Planta de Canadá.

MA 256673 (con 3 duplicados), fl: [CANADÁ] «Stachys / de Nutka [13 a 28-VIII-1791] / Neé iter» [et. ant.]; «Stachys / Nootka / Neé iter (Exp. Mal.)» [et. tra.]. Det. R. Morales, II2013. MA 774795, fl: [CANADÁ] «Ignoratur patria / 13 a 28VIII-1791] / Neé dedit» [et. ant.]; «Nee iter, 1789-1794 [et. tra.]». Det. J. L. Fern. Alonso, II-2013.

A esta planta corresponde la Stachys sylvatica L. indicada por Muñoz Garmendia (1992: 367), según cita Neé (1801: 271-272): «... en Monterey y Nootka el Oficial de marina D. Joseph Robredo y D. Manuel Esquerra Contador de la corbeta Atrevida. ... Tambien me traxéron los citados Señores de aquel sitio las siguientes plantas: ..., Stachys silvatica ...»

22.5. Stachys aff. mexicana Benth., Lab. Gen. Sp.: 541 (1834). Planta de México.

MA 256682, fl: [MÉXICO]«Stachys [III a XII-1791] / Neé dedit» [et. ant.]; «Stachys / Neé dedit» [et. tra.]. Det. J. L. Fern. Alonso, II-2013.

22.6. Stachys rigida Nutt. ex Benth. in DC., Prodr. 12: 472 (1848). Planta de México.

MA 256676, fl: [MÉXICO]«Stachys / DAS / Neé iter» [et. ant.]; «Stachys / DHS / Neé iter (Exp. Mal.)?» [et. tra.]. Det. J. L. Fern. Alonso, II-2013. MA 256677, fl: [MÉXICO]«Stachys / De / Neé iter» [et. ant.]; «Stachys / Neé iter (Exp. Mal.)?» [et. tra.]. Det. J. L. Fern. Alonso, II-2013. MA 256679, fl, fr: [MÉXICO]«Stachys / Neé iter» [et. ant.]; «Stachys / Neé iter (Exp. Mal..)?» [et. tra.]. Det. J. L. Fern. Alonso, II-2013. MA 256685, fr: [MÉXICO] «Stachys / ex Nova Hispania [III a XII-1791] / Neé iter» [et. ant.]; «Stachys / Nueva España Neé iter (Exp. Mal.)» [et. tra.]. Det. J. L. Fern. Alonso, I-2013. MA 256686, fl: [MÉXICO]«Stachys / Neé iter» [et. ant.]; «Stachys / Neé iter (Exp. Mal.?)» [et. tra.]. Det. J. L. Fern. Alonso, II-2013.

22.7. Stachys sericea Cav., Descr. P1. 1: 75 (1802). Fig. 2B. Planta de Chile.

Stachys candidissima Phil.; Stachys glabrata Phil. Ind. loc.: «Se cría en Talcaguano, donde la encontró Don Luis Neé.»; MA 103425, fl, fr: [CHILE] «Stachys sericea Curso no 182 Cav. / ex Talcahuano» [XI-1793] [et. ant. 1]; «Stachys sericea / Talcahuano en Chile / Neé dedit» [et. ant. 2]; «Horti Botanici Matritensis / Stachys sericea Cav. / Talcahuano en Chile / Neé dedit 103425 / Type of S. sericea Cav. (stored under name)» [et. tra.]. Det. J. L. Fern. Alonso, 1990. 
Garilleti (1993: 188) no encontró pliegos de Neé correspondientes a esta especie en MA.

22.8. Stachys truncata Kunze ex Benth., Lab. Gen. Sp.: 550 (1834). Planta de Chile.

MA 256689, fl, fr (2 duplicados): [CHILE] «Stachys / ex Talcahuano [XI-1793] / Neé iter» [et. ant.]; «Stachys / Talcahuano / Neé iter (Exp. Mal.)» [et. tra.]. Det. J. L. Fern. Alonso, II-2013.

23. Teucrium L., consta de unas 250 especies que viven en las regiones templadas y frías de todo el mundo, y más raramente en las regiones tropicales. Su mayor centro de diversidad es la región mediterránea (Cantino in Harley et al. 2004: 201). Cuatro especies en la colección Malaspina, 3 neotropicales y una australiana.

23.1. Teucrium bicolor Sm. in Rees, Cyclop. 35: n. 25 (1819). Planta americana.

Teucrium heterophyllum Cav., Icon. 6: 56, tab. 577 (I a V1801) nom illeg., non T. heterophyllum L'Hér.» [Ind. loc.: «Habitat in Talcahuano urbis Chilensis ditione, ibique Mimulus luteus, Hedyotis filiformis, Convolvulus hermanniae, Buttneria cordata, Colletia. Florent Novembri et Decembri. Vidi siccas.». Colector: L. Neé. Typus: LINN-Smith 995.25.»]. Teucrium cavanillesii Bert. ex Steud.; Teucrium chilense Desf. ex Steud.; Teucrium orchideum Lindl. Ic.: E. García Guillén \& F. Muñoz Garmendia, 2001: 124, lámina 58. Sign. RJB, VI, lám. 60. Chile: Talcahuano (Muñoz Garmendia 1992: 372). MA 256789, fr:: [PERÚ] «Teucrium heterophyllum / Cav, Icon / Ex Peruvia [V a IX-1790]» [et. ant.]; atribuida a Neé. Det. R. Morales, VIII2012. MA 256790, veget.: [PERÚ] «Teucrium heterophyllum / Perú [V a IX-1790] / Neé dedit» [et. ant.]; «Teucrium heterophyllum? / Perú / Cavanilles / Neé dedit (E.M.)» [et. ant.]. Det. R. Morales, VIII-2012. MA 256904, veget.: [PERÚ] «Teucrium / del Perú [V a IX-1790] / Neé iter» [et. ant.]; «Teucrium / del Perú / Neé iter (E.M.)» [et. tra.]. Det. R. Morales, VIII-2012. MA 256912, fl, fr: [CHILE] «Teucrium / Talcahuano [XI-1793]» [et. ant., atribuida a Neé]; «Teucrium / Talcahuano / (Neé iter (E.M.)). Det. R. Morales, VIII-2012. MA 256791: [CHILE] Teucrium heterophy / llum Cav. Ic. 5 ta. 5 / Neé dedit anno 1801. MA 256792: [CHILE] Teucrium heterophyllum/Icon Tab. 577. / Chile. / Neé dedit» [material tipo de T. heterophyllum Cav.]. MA 256793: [CHILE] Teucrium heterophy- / llum Ic. tab. 577 / Talcahuano y Con- / cepcion de Chile. [XI a XII-1793] / Neé dedit.» [material tipo de T. heterophyllum Cav.]. MA 256794 (2 pliegos) fl, fr: [ARGENTINA] «Teucrium heterophyllum / Cav. Ic. / De la punta de San Luis, / en las Pampas de Buenos Ayres [29-III-1794] / Neé iter» [et. ant.]; «Teucrium heterophyllum / Cav. Ic. / De la punta de San Luis, / en las Pampas de Buenos Ayres / Neé iter (E.M.)» [et. tra.]. Det. R. Morales VIII-2012. MA 256795 fl: [CHILE?] «Teucrium heterophyllum / Cavans. icon. tab. / Neé iter» [et. ant.]; «Teucrium heterophyllum Cav. / Neé iter,» [et. tra.]. Det. R. Morales, VIII-2012.
Garilleti (1993: 188) no encontró pliegos en el herbario MA, referidos a esta especie.

23.2. Teucrium cubense Jacq., Enum. Syst. Pl.: 25 (1760) subsp. cordobense Epling, Ann. Missouri Bot. Gard. 12: 113, p 8 (1925). Planta de Sudamérica.

Melosmon cubense (Jacq.) Small.; Teucrium chamaedrifolium Mill.; T. grisebachii Hieron.; T. laevigatum Vahl; MA 256905, fr: [ARGENTINA?] «Teucrium / Neé iter» [et. ant.]; «Teucrium / Neé iter (E.M.)» [et. tra.]. Det. R. Morales, VIII2012. MA $256908 \mathrm{fl}$, fr: [ARGENTINA] «Teucrium / del cerro de las Pampas de Buenos-Ayres. / Neé dedit» [et. ant.]; «Teucrium / del cerro de las Pampas de Buenos-Ayres. / Neé dedit (E. M.)» [et. tra.]. Det. R. Morales \& J. L. Fern. Alonso, VIII2012. MA 256914 (2 pliegos) fr: [ARGENTINA] «Teucrium / De la Punta de Sn. Luis en / las Pampas de Buenos ayres [29III-1794] / Neé iter» [et. ant.]; «Teucrium / De la Punta de Sn. Luis en / las Pampas de Buenos ayres / Neé iter (E. M.)» [et. tra.]. Det. R. Morales, VIII-2012.

23.3. Teucrium vesicarium Mill., Gard. Dict., ed. 8: n 17 (1768). Planta neotropical.

T. carthaginense Lange; T. hirtum Willd. ex Spreng.; T. inflatum Sw.; T. palustre Kunth; T. picardae Krug \& Urb.; T. tenuipes Epling; MA 256906, fl: [MÉXICO] «Teucrium / ex nov. Hispania / Neé iter. MA 256907 fl, fr: [PANAMÁ?] «Teucrium / ex Peruvia et Panamaide / Neé iter» [et. ant.]; «Teucrium / ex Peruvia et Panamaide / Neé iter» [et. tra.]. Det. R. Morales VIII-2012.

\subsection{Teucrium sp.}

MA 255423B (pro parte) fl: [AUSTRALIA] «Ocimum / Bahia Botanica / Neé dedit» [et. ant.]; «Ocymum / Bahia Botanica [III a IV-1793] / Cavanilles 7 Neé dedit (Exp. Mal.)» [et. tra.]. Det. J. L. Fern. Alonso \& R. Morales, II-2013.

24. Westringia Sm., consta de unas 25 especies exclusivas de Australia (Conn in Harley et al., 2004: 209). Una especie australina, se encuentra entre las recolecciones de la expedición.

24.1. Westringia fruticosa (Willd.) Druce, Rep. Bot. Exch. Cl. Brit. Isles 1916, Suppl. 2: 652 (1917).

Cunila fruticosa Willd., Sp. Pl. 1: 222 (1797). MA 257012, est: [AUSTRALIA] «Westringia rosmariniformis Act. Holmiens. / Ex Nova Hollandia. [III a IV-1793] / Neé dedit 1801.» [et. ant.]; «Westringia rosmariniformis Act. Holmiens. / Nueva Holanda / año 1801 / Neé iter» [et. tra.]. Det. R. Morales, II-2013. Hay un duplicado sin etiquetas ant.s. MA 257014, fl: [AUSTRALIA] «De esta se envió a Ventenat con el n ${ }^{0} 32$. / a Dresde con el n ${ }^{0}$ 173/ Neé iter /» [otra letra] arbuscula difussa / B B [Bahia Bo- 
tánica] » [III a IV-1793] / Westringia rosmarinifolia Schmith» [et. ant.s]; «Westringia rosmariniformis Smith / Neé iter (Exp. Mal.)?» [et. tra.]. Det. R. Morales, II-2013.

\section{AGRADECIMIENTOS}

Este trabajo se ha realizado como parte del proyecto CSD200800077 (2009-2013), que lleva por título «Expedición de circunnavega- ción Malaspina 2010: Cambio global y exploración de la biodiversidad del océano global. Bloque 8: La expedición Malaspina, ciencia y política allende los mares.» También ha contado con el apoyo del proyecto CGL 2010-19747 de «Estudios taxonómicos en plantas vasculares neotropicales», financiado por el Ministerio de Ciencia e Innovación de España. Agradecemos a Felix Muñoz Garmendia, su oportuna ayuda con variada información referente al desarrollo de la expedición Malaspina y al personal del herbario, biblioteca y archivo del Real Jardin Botánico por su permanente apoyo.

\section{BIBLIOGRAFÍA}

AmaYA, J. A. 2005. Mutis, apóstol de Linneo. 2 vols. Instituto Colombiano de Antropología e Historia. Bogotá.

Díaz Piedrahita, S. 2012. Parangón entre dos boticas granadinas. Rev. Acad. Colomb. Ci. 36(139): 219-235.

Díaz Piedrahita, S. \& Mantilla, L. C. 2002. La terapéutica en el Nuevo Reino de Granada. Un recetario franciscano del siglo XVIII. Academia Colombiana de Ciencias Exactas, Físicas y Naturales, Bogotá, D.C.

Epling, C. \& Játiva, C. 1964. Revisión el género Satureja en América del Sur. Brittonia 16(4): 393-416.

EpLing, C. 1949. Revisión el género Hyptis (Labiatae). Rev. Mus. La Plata, Secc. Bot. 7: 153-497.

Fernández Alonso, J. L. 2002. Estudios en Labiatae de Colombia III. Novedades en Lepechinia, Salvia y Satureja de Colombia. Anales Jard. Bot. Madrid 59(2): 344-348.

Fernández Alonso, J. L. 2010. Una nueva especie de Hyptis (Labiatae) de Colombia. Anales Jard. Bot. Madrid 67(2): 127-135.

FernáNDEZ Alonso, J. L. \& AMAYA, J. A. 1991. Historia del nombre genérico Escallonia Mutis ex L. f. (Saxifragaceae). Caldasia 16(78): 317-325.

Fernández Alonso, J. L. \& Rivera Díaz, 0. 2006. Labiatae. En: N. García \& G. Galeano (Eds.), Libro Rojo de Plantas de Colombia, 3: 385-582. Instituto Alexander von Humboldt, Instituto de Ciencias Naturales de la Universidad Nacional de Colombia, Bogotá.

García Guillén, E. \& MuÑoz Garmendia, F. 2001. VI Catálogo de dibujos de la expedición Malaspina del Real Jardín Botánico: sección de fondo Luis Neé. En: M. P. San Pío \& M. D. Higueras, La armonía natural. La naturaleza en la expedición marítima de Malaspina y Bustamante (17891794). Real Jardín Botánico de Madrid, Lunwerg \& Caja Madrid, Madrid.

GarilLeti, R. 1993. Herbarium cavanillesianum. Fontqueria 38: 1-249.

Harley, R. M.; Atrins, S.; Budantsev, A. L.; Cantino, P. D.; Conn, B. J.; Grayer, R.; Harley, M. M.; De KoK, R.; Kres- tovskaja, T.; Morales, R.; Paton, A. J.; Ryding, O. \& UpSON, T. 2004. Labiatae. En: J. W. Kadereit (Ed.), The families and genera of vascular plants VII. Flowering plants. Dicotyledons: Lamiales (except Acanthaceae including Avicenniaceae): 167-275. Springer, Berlin.

HARLEY, R. \& PASTORE, J. 2012. A generic revision and new combinations in the Hyptidinae (Lamiaceae), based on molecular and morphological evidence. Phytotaxa 58: 1-55.

IBaÑEZ Montoya, M. V. 1992. La expedición Malaspina 17891794. Tomo IV. Trabajos científicos y correspondencia de Tadeo Haenke. Ministerio de Defensa, Museo Naval \& Lunwerg Editores, Madrid.

Keng, H. 1978. Labiatae. Flora Malesiana ser. 1, 8(3): 301394.

Morales, R. 2010. Labiadas de España en América. Intercambio de usos. En: M. L. Pochettino, A. H. Ladio \& P. Arenas (Eds.), Tradiciones y transformaciones en Etnobotánica: 384-393.

MuÑoz Garmendia, F. 1992. La expedición Malaspina 17891794. Tomo III. Diarios y trabajos botánicos de Luis Neé. Ministerio de Defensa, Museo Naval \& Lunwerg Editores. Madrid.

MuÑoz Garmendia, F. 2001. La botánica y los botánicos de la expedición Malaspina, Luis Neé y Tadeo Haenke. En: P. San Pio (Ed.), La armonía natural. La naturaleza en la expedición marítima de Malaspina y Bustamante (1789-1794): 63-82. Lunwerg Editores, Madrid.

NeÉ, L. 1801. Descripción de varias especies nuevas de encina (Quercus de Linneo). Por D. Luis Neé. Anales de Ciencias Naturales 3(9): 261-278.

Pelser, P. B.; Barcelona, J. F. \& Nickrent, D. L. (Eds.) 2011 onwards. Co's Digital Flora of the Philippines. www.philippineplants.org.

PresL, K. B. 1825-1835. Reliquiae Hankeanae seu descriptiones et icones... I (1825-1830). II (1831-1835). Pragae.

Schmidt-Lebuhn, A. N. 2008. A revisión of the genus Minthostachys (Labiatae). Mem. New York Bot. Gard. 98: 1-77. 